\title{
Non-homogeneous boundary value problems for the Korteweg-de Vries and the Korteweg-de Vries-Burgers equations in a quarter plane
}

\author{
Jerry L. Bona ${ }^{\mathrm{a}, *}$, S.M. Sun ${ }^{\mathrm{b}}$, Bing-Yu Zhang ${ }^{\mathrm{c}}$ \\ a Department of Mathematics, Statistics \& Computer Science, University of Illinois at Chicago, Chicago, IL 60607, USA \\ $\mathrm{b}$ Department of Mathematics, Virginia Polytechnic Institute and State University, Blacksburg, VA 24061-4097, USA \\ ${ }^{\mathrm{c}}$ Department of Mathematical Sciences, University of Cincinnati, Cincinnati, OH 45221-0025, USA
}

Received 28 April 2006; received in revised form 2 May 2007; accepted 22 July 2007

Available online 24 January 2008

\begin{abstract}

$$
\left.\begin{array}{l}
u_{t}+u_{x}+u u_{x}+u_{x x x}=0, \quad \text { for } x, t \geqslant 0, \\
u(x, 0)=\phi(x), \quad u(0, t)=h(t)
\end{array}\right\}
$$
\end{abstract}

Attention is given to the initial-boundary-value problems (IBVPs)

for the Korteweg-de Vries (KdV) equation and

$$
\left.\begin{array}{l}
u_{t}+u_{x}+u u_{x}-u_{x x}+u_{x x x}=0, \quad \text { for } x, t \geqslant 0, \\
u(x, 0)=\phi(x), \quad u(0, t)=h(t)
\end{array}\right\}
$$

for the Korteweg-de Vries-Burgers (KdV-B) equation. These types of problems arise in modeling waves generated by a wavemaker in a channel and waves incoming from deep water into near-shore zones (see [B. Boczar-Karakiewicz, J.L. Bona, Wave dominated shelves: a model of sand ridge formation by progressive infragravity waves, in: R.J. Knight, J.R. McLean (Eds.), Shelf Sands and Sandstones, in: Canadian Society of Petroleum Geologists Memoir, vol. 11, 1986, pp. 163-179] and [J.L. Bona, W.G. Pritchard, L.R. Scott, An evaluation of a model equation for water waves, Philos. Trans. Roy. Soc. London Ser. A 302 (1981) 457-510] for example). Our concern here is with the mathematical theory appertaining to these problems. Improving upon the existing results for $(0.2)$, we show this problem to be (locally) well-posed in $H^{s}\left(\Re^{+}\right)$when the auxiliary data $(\phi, h)$ is $\operatorname{drawn}$ from $H^{s}\left(\Re^{+}\right) \times$ $H_{\text {loc }}^{\frac{s+1}{3}}\left(\Re^{+}\right)$, provided only that $s>-1$ and $s \neq 3 m+\frac{1}{2}(m=0,1,2, \ldots)$. A similar result is established for $(0.1)$ in $H_{v}^{s}\left(\Re^{+}\right)$ provided $(\phi, h)$ lies in the space $H_{v}^{s}\left(\Re^{+}\right) \times H_{\text {loc }}^{\frac{s+1}{3}}\left(\Re^{+}\right)$. Here, $H_{v}^{s}\left(\Re^{+}\right)$is the weighted Sobolev space

$$
H_{v}^{S}\left(\Re^{+}\right)=\left\{f \in H^{S}\left(\Re^{+}\right) ; e^{v x} f \in H^{s}\left(\Re^{+}\right)\right\}
$$

with the obvious norm (cf. Kato [T. Kato, On the Cauchy problem for the (generalized) Korteweg-de Vries equations, in: Advances in Mathematics Supplementary Studies, in: Studies Appl. Math., vol. 8, 1983, pp. 93-128]). Both local and global in time results are derived. An added outcome of our analysis is a very strong smoothing property associated with the problems

\footnotetext{
* Corresponding author.

E-mail addresses: bona@math.uic.edu (J.L. Bona), sun@math.vt.edu (S.M. Sun), bzhang@math.uc.edu (B.-Y. Zhang).
} 
(0.1) and (0.2) which may be expressed as follows. Suppose $h \in H_{\mathrm{loc}}^{\infty}$ and that for some $v>0$ and $s>-1$ with $s \neq 3 m+\frac{1}{2}$ $(m=0,1,2, \ldots), \phi$ lies in $H_{v}^{s}\left(\Re^{+}\right)$(respectively $H^{s}\left(\Re^{+}\right)$). Then the corresponding solution $u$ of the IBVP $(0.1)$ (respectively the IBVP (0.2)) belongs to the space $C\left(0, \infty ; H_{\nu}^{\infty}\left(\Re^{+}\right)\right)$(respectively $C\left(0, \infty ; H^{\infty}\left(\Re^{+}\right)\right)$). In particular, for any $s>-1$ with $s \neq 3 m+\frac{1}{2}(m=0,1,2, \ldots)$, if $\phi \in H^{s}\left(\Re^{+}\right)$has compact support and $h \in H_{\mathrm{loc}}^{\infty}\left(\Re^{+}\right)$, then the IBVP $(0.1)$ has a unique solution lying in the space $C\left(0, \infty ; H^{\infty}\left(\Re^{+}\right)\right)$.

(c) 2008 Elsevier Masson SAS. All rights reserved.

Keywords: Korteweg-de Vries equation; Korteweg-de Vries-Burgers equation; Initial-boundary-value problems; Nonlinear dispersive wave equations

\section{Introduction}

In this paper, we continue the study of the initial-boundary-value problem (IBVP) for the Korteweg-de Vries (KdV) equation and the Korteweg-de Vries-Burgers (KdV-B) equation posed in a quarter plane, namely

$$
\left.\begin{array}{l}
u_{t}+u_{x}+u u_{x}-\mu u_{x x}+u_{x x x}=0, \quad \text { for } x, t \geqslant 0, \\
u(x, 0)=\phi(x), \quad u(0, t)=h(t),
\end{array}\right\}
$$

where $\mu=0$ for KdV and $\mu>0$ for KdV-B. These IBVP's arise in mathematical descriptions of water waves generated by a wave maker in a channel and in other situations where a wavetrain is generated at or impinges upon one end of a stretch of a medium of propagation that suffers both non-linear and dispersive effects in response to disturbances (see $[2,3,5,35])$. Our main concern is the well-posedness of (1.1) in the sense to be specified now, in the classical Sobolev spaces $H^{s}\left(\Re^{+}\right)$for small values of $s$.

Definition 1.1 (Well-posedness). For a given real number $s$, the IBVP (1.1) is said to be well-posed in the space $H^{s}\left(\Re^{+}\right) \times H_{\text {loc }}^{\frac{s+1}{3}}\left(\Re^{+}\right)$if for any $r>0$, there exists a $T=T(r)>0$ depending only on $s$ and $r$ such that for $s$-compatible $(\phi, h) \in H^{s}\left(\Re^{+}\right) \times H^{\frac{s+1}{3}}(0, T)$ satisfying

$$
\|(\phi, h)\|_{H^{s}\left(\Re^{+}\right) \times H^{\frac{s+1}{3}}(0, T)} \leqslant r
$$

the IBVP (1.1) admits a unique solution

$$
u \in C\left([0, T] ; H^{s}\left(\Re^{+}\right)\right) .
$$

Moreover, the solution depends continuously in this latter space on variations of the auxiliary data in their respective function spaces.

A pair $(\phi, h) \in H^{s}\left(\Re^{+}\right) \times H^{\frac{s+1}{3}}(0, T)$ is said to be $s$-compatible for (1.1) if, in case $s>\frac{1}{2}$,

$$
\phi_{k}(0)=h_{k}(0)
$$

for $k=0,1, \ldots,[s / 3]-1$ when $s-3[s / 3] \leqslant \frac{1}{2}$ and for $k=0,1, \ldots,[s / 3]$ when $s-3[s / 3]>\frac{1}{2}$, where $h_{k}(t) \equiv h^{(k)}(t)$ is the $k$ th order derivative of $h$,

$$
\left.\begin{array}{l}
\phi_{0}(x)=\phi(x), \quad \text { and } \\
\phi_{k}(x)=-\left(\phi_{k-1}^{\prime \prime \prime}(x)+\phi_{k-1}^{\prime}(x)-\mu \phi_{k-1}^{\prime \prime}(x)+\sum_{j=0}^{k-1}\left[\phi_{j}(x) \phi_{k-j-1}(x)\right]^{\prime}\right)
\end{array}\right\}
$$

for $k=1,2, \ldots$ Here, for non-negative numbers $r,[r]$ is the largest integer less than or equal to $r$.

The well-posedness described by Definition 1.1 is usually called local well-posedness since the life-span $T$ of the solution depends on the size $r$ of the auxiliary data. If, instead, $T$ is independent of $r$, then (1.1) is said to be globally well-posed in the space $H^{s}\left(\Re^{+}\right) \times H_{\text {loc }}^{\frac{s+1}{3}}\left(\Re^{+}\right)$. When $s \geqslant 3$, the term 'solution' in Definition 1.1 is understood to refer to a strong solution, which is to say $u$ satisfies the equation in (1.1) in the space $L^{2}\left(\Re^{+}\right)$for all $t \in[0, T]$. When $s<3$, the solution $u$ in Definition 1.1 is understood to be a mild solution as defined below in Definition 1.2. One of the advantages of using the concept of mild solution instead of solution in the sense of distributions is that one does not need to be concerned with whether or not the non-linear term $u u_{x}$ in the equation makes sense in the relevant function class, a point that is especially telling when $s$ is negative. 
Definition 1.2 (mild solution). Let $s \in \Re$ and $T>0$ be given. For a given $s$-compatible pair $(\phi, h) \in H^{s}\left(\Re^{+}\right) \times$ $H_{\text {loc }}^{\frac{s+1}{3}}\left(\Re^{+}\right)$, a function $u \in C\left([0, T] ; H^{s}\left(\Re^{+}\right)\right)$is said to be a mild solution of the IBVP $(1.1)$ on the time interval $[0, T]$ if there exists a sequence $\left\{u_{n}\right\}_{n=1}^{\infty}$ in the space

$$
C\left([0, T] ; H^{3}\left(\Re^{+}\right)\right) \cap C^{1}\left([0, T] ; L^{2}\left(\Re^{+}\right)\right)
$$

with

$$
\phi_{n}(x)=u_{n}(x, 0), \quad h_{n}(t)=u_{n}(0, t), \quad n=1,2, \ldots,
$$

such that

(i) $u_{n}$ solves the equation in $(1.1)$ in $L^{2}\left(\Re^{+}\right)$for $0<t<T$;

(ii) $\lim _{n \rightarrow \infty}\left\|u_{n}-u\right\|_{C\left([0, T] ; H^{s}\left(\Re^{+}\right)\right)}=0$;

(iii) $\lim _{n \rightarrow \infty}\left\|\phi_{n}-\phi\right\|_{H^{s}\left(\Re^{+}\right)}=0$ and $\lim _{n \rightarrow \infty}\left\|h_{n}-h\right\|_{H^{\frac{s+1}{3}}(0, T)}=0$.

Remark. If $s \geqslant 3$ and $u$ is a strong solution of (1.1), then the constant sequence $u_{n}=u$ for all $n$ suffices to determine $u$ as a mild solution.

Boundary-value problems for non-linear, dispersive wave equations began with the work of Bona and Bryant [3] for the BBM-equation. The study of the IBVP (1.1) with $\mu=0$ was initiated by Ton in [54], where existence and uniqueness were established assuming that the initial data $\phi$ is smooth and the boundary data $h \equiv 0$. The first wellposedness result in the sense of Definition 1.1 for the IBVP (1.1) was presented by Bona and Winther [13,14]; they showed that the IBVP $(1.1)$ is (globally) well-posed in the space $H^{3 k+1}\left(\Re^{+}\right)$with $(\phi, h) \in H^{3 k+1}\left(\Re^{+}\right) \times H_{\text {loc }}^{k+1}\left(\Re^{+}\right)$ for $k=1,2, \ldots$ There have been many works on (1.1) since then. The reader is referred to [4,8-12,20,26-29,33, $32,31,30,34]$ and the references therein for an overall literature review. In particular Bona, Sun and Zhang in [8] extended the theory of Kenig, Ponce and Vega in [41,42] on the initial value problem (IVP) for the KdV equation posed on the whole line $\Re$ to the IBVP (1.1), showing that it is well-posed in the space $H^{s}\left(\Re^{+}\right) \times H_{\text {loc }}^{\frac{s+1}{3}}\left(\Re^{+}\right)$for $s>\frac{3}{4}$. In [20], Colliander and Kenig demonstrated how the powerful theory developed by Kenig, Ponce and Vega, Bourgain and others for the pure initial value problems for non-linear dispersive wave equations can be adapted to deal with initial boundary value problems for the same equations. They showed in [20] that for a given $s$-compatible pair $(\phi, h) \in H^{s}\left(\Re^{+}\right) \times H_{\mathrm{loc}}^{\frac{s+1}{3}}\left(\Re^{+}\right)$with $0 \leqslant s \leqslant 1, s \neq \frac{1}{2}$, the IBVP $(1.1)$ admits a solution $u \in C\left([0, T] ; H^{s}\left(\Re^{+}\right)\right)$which depends continuously on $(\phi, h)$. This result was strengthened later by Holmer [34] to include the case $-\frac{3}{4}<s<0$. In a recent paper [12], Bona, Sun and Zhang showed that the IBVP (1.1) possesses a strong global smoothing property that comes about because of the dissipative mechanism introduced through imposition of the boundary condition at $x=0$. With the aid of this boundary smoothing property and the use of restricted Bourgain spaces, they resolved the uniqueness issue left open in [20] and showed that the IBVP (1.1) is unconditionally well-posed in the space $H^{s}\left(\Re^{+}\right) \times H_{\mathrm{loc}}^{\frac{s+1}{3}}\left(\Re^{+}\right)$for any $s>-\frac{3}{4}$.

The following question then arises naturally.

Question 1.3. Is the IBVP (1.1) well-posed in the space $H^{s}$ for any values of $s<-\frac{3}{4}$ ?

The issue of how large can be the spaces of auxiliary data and still maintain well-posedness also arises for the pure initial value problem (IVP) for the KdV-equation posed on the whole line $\Re$, viz.

$$
\left.\begin{array}{l}
u_{t}+u u_{x}+u_{x x x}=0, \quad x, t \in \Re \\
u(x, 0)=\phi(x),
\end{array}\right\}
$$

or posed with periodic boundary conditions, which is to say, posed on the unit circle $S$ in the plane,

$$
\left.\begin{array}{ll}
u_{t}+u u_{x}+u_{x x x}=0, & x \in S, t \in \Re \\
u(x, 0)=\phi(x), & x \in S .
\end{array}\right\}
$$


After considerable effort (see [7,6,15,17-19,21,22,37,39-44,46,50,51,53] and the references contained therein), it is understood that the IVP (1.3) is well-posed in the space $H^{s}(\Re)$ for $s>-\frac{3}{4}$ and the IVP (1.4) is well-posed in the space $H^{s}(S)$ for $s \geqslant-\frac{1}{2}$. The analogue of Question 1.3 in the context of (1.3)-(1.4) is the following.

Question 1.4. Is the IVP (1.3) well-posed in $H^{s}(\Re)$ for some $s<-\frac{3}{4}$ or is the IVP (1.4) well-posed in $H^{s}(S)$ for some $s<-\frac{1}{2}$ ?

The answer is instructive. For the IVP (1.3), when $s<-\frac{3}{4}$, the IVP (1.3) has been shown to be ill-posed in $H^{s}(\Re)$ in the sense that the solution map, if it were to exist, cannot be locally uniformly continuous (see $[16,55,19,45])$. The same can be said for the IVP (1.4); it is ill-posed in $H^{s}(S)$ when $s<-\frac{1}{2}$ in the sense that the solution map cannot be locally uniformly continuous. When $s=-\frac{3}{4}$, a weaker form of local well-posedness has been established for (1.3) in [21]. Thus, the indications were that the answer to Question 1.4 was almost certainly negative. It came as quite a surprise when Kappeler and Topalov [36] demonstrated recently that the IVP (1.4) is (globally) well-posed in the space $H^{s}(S)$ for $s \geqslant-1$. In addition, there is another recent and very interesting paper [47] of Molinet and Ribaud on the pure initial-value problem

$$
\left.\begin{array}{ll}
u_{t}+u u_{x}+u_{x x x}-u_{x x}=0, & x \in \Re, t>0 \\
u(x, 0)=\psi(x), & x \in \Re
\end{array}\right\}
$$

for the KdV-Burgers equation. They showed that (1.5) is well-posed in the space $H^{s}(\Re)$ for $s>-1$ and is ill-posed when $s<-1$ in the sense that the corresponding solution map is not $C^{2}$. This is also a surprising result since the pure initial-value problem for the Burgers equation, namely

$$
\left.\begin{array}{ll}
u_{t}+u u_{x}-u_{x x}=0, & x \in \Re, t>0 \\
u(x, 0)=\psi(x), & x \in \Re,
\end{array}\right\}
$$

is known to be well-posed in the space $H^{s}(\Re)$ for $s \geqslant-\frac{1}{2}$ and is ill-posed in $H^{s}(\Re)$ for any $s<-\frac{1}{2}$ [1,25]. Molinet and Ribaud achieved their result by taking full advantage of the combination of the dispersion introduced through the term $u_{x x x}$ and the dissipation inherent in the Burgers' term $-u_{x x}$, though their analysis is informed by the work of Bourgain, and Kenig, Ponce and Vega. The corresponding solution map is real analytic when $s>-1$. In contrast, the approach of Kappeler and Topalov is completely different from those of Bourgain and Kenig, Ponce and Vega, being based on the classical inverse scattering transform. The corresponding solution map associated with the IVP (1.4) is continuous, but not locally uniformly continuous when $s<-\frac{1}{2}$.

An implication of the work of Kappeler-Topalov and Molinet-Ribaud is that it is reasonable to conjecture the IBVP (1.1) is well-posed in the space $H^{s}\left(\Re^{+}\right)$for at least some range of $s<-\frac{3}{4}$. Indeed, their works not only indicate that an affirmative answer to Question 1.3 is possible, but also suggest two possible approaches:

(a) seeking to use the dissipative mechanism inherent in imposing the boundary condition for (1.1) at $x=0$ in a way reminiscent of what Molinet and Ribaud did with the Burgers term in (1.6);

(b) using the inverse scattering methodology as did Kappeler and Topalov.

Recent work in the direction of an inverse scattering transform for (1.1) by Fokas, Its and Pelloni [33,32,31,30] shows promise for its use in rigorous studies. In the present essay, we have elected approach (a), in part because results obtained by such considerations are likely to have more scope as they rely upon a less rigid structure. A theory based on the inverse scattering transform is being investigated separately.

There are two important auxiliary points arising in the present analysis that are worth singling out, as they have independent interest. One is an accurate appraisal of the damping implied by imposition of the boundary condition at $x=0$. The other is a relation between the IBVP's for the KdV-equation and the KdV-Burgers equation. These points are explained now.

It is a simple matter to see that the imposition of the boundary condition $u(0, t)=h(t)$ in (1.1) results in dissipation. Indeed, suppose for example that $h(t) \equiv 0$ for all $t \geqslant 0$ and that $u$ is a suitably smooth solution of (1.1) which, along 
with its first few partial derivatives, decays rapidly to zero as $x \rightarrow \infty$. Multiplying the KdV-equation by $u$, integrating over the half-line $\mathfrak{R}^{+}$, and integrating by parts, leads to the formula

$$
\frac{d}{d t} \int_{0}^{\infty}|u(x, t)|^{2} d x=-\frac{1}{2} u_{x}^{2}(0, t) \quad \text { for all } t \geqslant 0 .
$$

Thus the $L^{2}$-norm of the solution is seen to be decreasing, and strictly so whenever $u_{x}(0, t) \neq 0$. This dissipative mechanism is somewhat more subtle than that induced by a Burgers-type term in the equation itself, or other forms of point dissipation. These have been studied and quantified in the works of Bona, Sun and Zhang [12] and Russell and Zhang [48]. In particular, it was shown in [12] that the IBVP for the linear problem

$$
\left.\begin{array}{l}
u_{t}+u_{x}+u_{x x x}=0, \quad x, t>0, \\
u(x, 0)=0, \quad u(0, t)=h(t), \quad x, t>0
\end{array}\right\}
$$

associated to (1.1) has the following smoothing property; its solution $u(x, t)$ is the restriction to $\mathfrak{R}^{+} \times \mathfrak{R}^{+}$of a function $w(x, t)$ defined on $\Re \times \Re$ which is such that

$$
\left(\int_{-\infty}^{\infty} \int_{-\infty}^{\infty}(1+|\xi|)^{2 s}\left(1+\left|\tau-\xi^{3}\right|\right)^{2 b}|\hat{w}(\xi, \tau)|^{2} d \xi d \tau\right)^{1 / 2} \leqslant C\|h\|_{H^{\frac{3 b+s-1 / 2}{3}}\left(\Re^{+}\right)}
$$

where $b$ is any value in $\left[0, \frac{1}{2}-\frac{s}{3}\right.$ ) if $-\frac{3}{2} \leqslant s<1, b$ is any value in $\left[0, \frac{5}{6}-\frac{s}{3}\right]$ if $-\frac{1}{2}<s<1$ and $C$ is a constant depending only on $s$ and $b$. As a direct consequence of this estimate, we derive the corollary that

$$
h \in H_{\text {loc }}^{\frac{s+1}{3}}\left(\Re^{+}\right) \text {implies that the solution } u \text { of }(1.7) \text { belongs to the space } L^{2}\left(0, T ; H^{s+\frac{3}{2}}\left(\Re^{+}\right)\right) \text {. }
$$

This is a much stronger smoothing result than the well-known Kato smoothing property which only implies that $u \in L^{2}\left(0, T ; H_{\mathrm{loc}}^{s+1}\left(\Re^{+}\right)\right)$(see [12]). This global boundary smoothing property is the key to our proof in [12] that the IBVP (1.1) is unconditionally well-posed in $H^{s}\left(\Re^{+}\right)$for any $s>-\frac{3}{4}$.

We also mention that it is not only the imposition of a boundary condition at $x=0$ that can result in smoothing of solutions of (1.1). Indeed, for both the pure initial-value problem (1.3) and the initial-boundary-value problem for the KdV-equation ((1.1) with $\mu=0)$, more rapid decay of the initial value as $x \rightarrow+\infty$ leads to an increase in the smoothness of the solution for $t>0$. This well-known fact (see, e.g. [23,24,26,27,38,46,49]) has, for example, been exploited by Faminskii [26,27] and Kruzhkov and Faminskii [46] in their study of well-posedness issues for the KdV-equation.

Now, attention is directed to a connection between the $\mathrm{KdV}$ equation and the $\mathrm{KdV}$-Burgers equation. Let $\alpha, \beta \in \Re$ be given and consider the transformation

$$
u(x, t)=e^{\alpha x+\beta t} v(x, t) .
$$

A direct calculation shows that $u$ is a solution of the $\mathrm{KdV}$ equation

$$
u_{t}+u_{x}+u u_{x}+u_{x x x}=0
$$

if and only if $v$ is a solution of the equation

$$
v_{t}+\left(\alpha+\alpha^{3}+\beta\right) v+\left(3 \alpha^{2}+1\right) v_{x}+v_{x x x}+3 \alpha v_{x x}+e^{\beta t+\alpha x}\left(\alpha v^{2}+v v_{x}\right)=0 .
$$

If we choose

$$
\alpha<0, \quad \beta=-\alpha-\alpha^{3},
$$

then $v(x, t)$ is a solution of the IBVP for the KdV-Burgers type equation

$$
\left.\begin{array}{ll}
v_{t}+\left(3 \alpha^{3}+1\right) v_{x}+e^{-\left(\alpha+\alpha^{3}\right) t+\alpha x}\left(v v_{x}+\alpha v^{2}\right)+v_{x x x}+3 \alpha v_{x x}=0, & x, t>0, \\
v(x, 0)=e^{-\alpha x} \phi(x), & x \geqslant 0, \\
v(0, t)=e^{\left(\alpha+\alpha^{3}\right) t} h(t), & t \geqslant 0
\end{array}\right\}
$$


if and only if $u(x, t)$ is a solution of (1.1). Thus, one is led to consider the IBVP for the variable-coefficient KdVBurgers equation posed on the half line $\mathfrak{R}^{+}$, viz.

$$
\left.\begin{array}{ll}
v_{t}+\rho v_{x}+\gamma v v_{x}+a_{1}(x) b_{1}(t) v v_{x}+a_{2}(x) b_{2}(t) v^{2}+v_{x x x}-v_{x x}=0, & x, t>0, \\
v(x, 0)=\psi(x), & x \geqslant 0, \\
v(0, t)=g(t), & t \geqslant 0
\end{array}\right\}
$$

where $\rho>0$ and $\gamma \in R$ are constants, $a_{j} \in H^{\infty}[0, \infty)$ and $b_{j} \in C^{\infty}[0, \infty)$ for $j=1,2$. Note that well-posedness of (1.12) in the space $H^{s}\left(\Re^{+}\right)$, the $s$-compatibility of the initial value $\psi$ and the boundary value $g$, and the concept of mild solution of the IBVP (1.12) can be defined just as described in Definitions 1.1 and 1.2 for the IBVP (1.1). Using the approach developed in our earlier paper [12,56], we can extend Molinet and Ribaud's work [47] on the pure initial-value problem (1.5) to the IBVP (1.12) as follows.

Theorem 1.5 (local well-posedness). Let $s>-1$ be given with $s \neq 3 m+\frac{1}{2}, m=0,1,2, \ldots$ For any $r>0$, there exists a $T=T(r)>0$ such that if $(\psi, g) \in H^{s}\left(\Re^{+}\right) \times H^{\frac{s+1}{3}}(0, T)$ is s-compatible with respect to the equation in (1.12) and satisfies

$$
\|(\psi, g)\|_{H^{s}\left(\Re^{+}\right) \times H^{\frac{s+1}{3}}(0, T)}<r,
$$

then the IBVP (1.12) admits a unique solution $u \in C\left([0, T] ; H^{s}\left(\Re^{+}\right)\right)$. Moreover, the corresponding solution map is (real) analytic from the space $H^{s}\left(\Re^{+}\right) \times H^{\frac{s+1}{3}}(0, T)$ to the space $C\left([0, T] ; H^{s}\left(\Re^{+}\right)\right)$.

Theorem 1.5 is a local result since the asserted life span $(0, T)$ of the solution depends on the size $r$ of its initial and boundary data. As in [8], the following global well-posedness results obtain for the IBVP (1.12).

Theorem 1.6 (global well-posedness). Assume that the system (1.12) satisfies

$$
b_{2}(t) a_{2}(x)-\frac{1}{3} b_{1}(t) a_{1}^{\prime}(x) \equiv 0 \quad x, t \in(0,+\infty) .
$$

(i) Let $s \geqslant 0$ and $T>0$ be given with $s \neq 3 m+\frac{1}{2}, m=0,1,2, \ldots$ For any $s$-compatible pair

$$
(\psi, g) \in H^{s}\left(\Re^{+}\right) \times H^{\frac{s+1}{3}+\eta(s)}(0, T)
$$

where

$$
\eta(s)= \begin{cases}\epsilon>0, & \text { if } 0 \leqslant s<3, \\ 0, & \text { if } s \geqslant 3,\end{cases}
$$

the IBVP (1.12) admits a unique solution $u \in C\left([0, T] ; H^{s}\left(\Re^{+}\right)\right)$. Moreover, the corresponding solution map is (real) analytic from the space $H^{s}\left(\Re^{+}\right) \times H^{\frac{s+1}{3}+\eta(s)}(0, T)$ to the space $C\left([0, T] ; H^{s}\left(\Re^{+}\right)\right)$.

(ii) Let $s \in(-1,0)$ and $T>0$ be given. For any $(\psi, g) \in H^{s}\left(\Re^{+}\right) \times H^{\frac{1}{3}+\epsilon}(0, T)$, the IBVP $(1.12)$ admits a unique solution $u \in C\left([0, T] ; H^{s}\left(\Re^{+}\right)\right) \cap C\left((0, T] ; L^{2}\left(\Re^{+}\right)\right)$. Moreover, if $g \in H^{\infty}(0, T)$, then $u \in C\left((0, T] ; H^{\infty}\left(\Re^{+}\right)\right)$.

Introduce some natural weighted Sobolev-spaces following Kato [39]. For given $v>0$ and $s \in \Re$, let $H_{v}^{s}\left(\Re^{+}\right)$ denote the Hilbert space

$$
H_{v}^{s}\left(\Re^{+}\right) \equiv\left\{f \in H^{s}\left(\Re^{+}\right) ; e^{v x} f \in H^{s}\left(\Re^{+}\right)\right\}
$$

with the norm

$$
\|f\|_{H_{v}^{s}\left(\Re^{+}\right)} \equiv\left(\|f\|_{H^{s}\left(\Re^{+}\right)}^{2}+\left\|e^{\nu x} f\right\|_{H^{s}\left(\Re^{+}\right)}^{2}\right)^{\frac{1}{2}} .
$$

The following local well-posedness result for the IBVP (1.1) then follows as a corollary to Theorem 1.5. 
Theorem 1.7 (local well-posedness). Let $v>0$ and $s>-1$ be given with $s \neq 3 m+\frac{1}{2}, m=0,1,2, \ldots$ For any $r>0$, there exists a $T=T(r)>0$ such that if an s-compatible pair

$$
(\phi, h) \in H_{v}^{s}\left(\Re^{+}\right) \times H^{\frac{s+1}{3}}(0, T)
$$

satisfying

$$
\|(\phi, h)\|_{H_{v}^{s}\left(\Re^{+}\right) \times H^{\frac{s+1}{3}}(0, T)}<r
$$

is posed as auxiliary data, then the IBVP (1.1) admits a unique solution $u \in C\left([0, T] ; H_{v}^{s}\left(\Re^{+}\right)\right)$. Moreover, the corresponding solution map is (real) analytic from $H_{v}^{s}\left(\Re^{+}\right) \times H^{\frac{s+1}{3}}(0, T)$ to $C\left([0, T] ; H_{v}^{s}\left(\Re^{+}\right)\right)$.

In addition, the following global well-posedness result holds for the IBVP (1.1).

Theorem 1.8 (global well-posedness).

(i) Let $s \geqslant 0$ and $T>0$ be given with $s \neq 3 m+\frac{1}{2}, m=0,1,2, \ldots$ For any $(\phi, h) \in H_{v}^{s}\left(\Re^{+}\right) \times H^{\frac{s+1}{3}+\eta(s)}(0, T)$, the IBVP (1.1) admits a unique solution

$$
u \in C\left([0, T] ; H_{v}^{s}\left(\Re^{+}\right)\right) .
$$

Moreover, the corresponding solution map is (real) analytic from the space $H_{v}^{s}\left(\Re^{+}\right) \times H^{\frac{s+1}{3}+\eta(s)}(0, T)$ to the space $C\left([0, T] ; H_{v}^{s}\left(\Re^{+}\right)\right)$.

(ii) Let $s \in(-1,0), v>0$ and $T>0$ be given. For $\epsilon>0$ and any $(\phi, h) \in H_{v}^{s}\left(\Re^{+}\right) \times H^{\frac{1}{3}+\epsilon}(0, T)$, the IBVP (1.1) admits a unique solution

$$
u \in C\left([0, T] ; H_{v}^{s}\left(\Re^{+}\right)\right) \cap C\left((0, T] ; L_{v}^{2}\left(\Re^{+}\right)\right) .
$$

Moreover, if $h \in H^{\infty}(0, T)$, then $u \in C\left((0, T] ; H_{v}^{\infty}\left(\Re^{+}\right)\right)$.

Note that Theorem 1.8 does not follow directly from Theorem 1.6 since system (1.11) does not satisfy assumption (1.13). However, (1.11) can be rewritten in the form

$$
\left.\begin{array}{ll}
v_{t}+\left(3 \alpha^{3}+1\right) v_{x}+\frac{1}{2} \alpha u v+\frac{1}{2}(u v)_{x}+v_{x x x}+3 \alpha v_{x x}=0, & x, t>0, \\
v(x, 0)=e^{-\alpha x} \phi(x), & x \geqslant 0, \\
v(0, t)=e^{\left(\alpha+\alpha^{3}\right) t} h(t), & t \geqslant 0
\end{array}\right\}
$$

where $u=u(x, t)$ is the solution of $(1.1)$, which is known (cf. [12,29]) to exist globally in the space $H^{s}\left(\Re^{+}\right)$if $(\phi, h) \in H^{s}\left(\Re^{+}\right) \times H_{\text {loc }}^{\frac{s+1}{3}+\eta(\epsilon)}\left(\Re^{+}\right)$is $s$-compatible with respect to the system (1.1). Since the equation in (1.14) is a linear equation with a variable coefficient $u=u(x, t)$ that exists globally in the space $H^{s}\left(\Re^{+}\right)$, the solution $v$ of (1.14) exists globally in the space $H^{s}\left(\Re^{+}\right)$, from which Theorem 1.8 follows.

The paper is organized as follows. In Section 2, explicit representation formulas for solutions of initial-boundaryvalue problems for the linear KdV-Burgers equation are presented. These are developed along the same lines as those in our earlier paper [8]. Various estimates will be established for the linear problems associated to (1.1) and (1.12) and these play a central role in the analysis of the non-linear problems. In Section 3, the well-posedness results for the IBVP (1.1) and (1.12) as described in Theorems 1.5-1.8 are established. The last section is an Appendix A containing explanations of some technical lemmas that are central to the analysis in Sections 2 and 3.

\section{Linear problems}

This section is divided into two subsections. In the first, consideration is given to linear problems associated to the $\mathrm{KdV}-$ Burgers equation. As mentioned above, explicit representation formulas for solutions of initial-boundary-value problems for this equation will be derived. Then, the boundary integral operator that arises in the solution formulas will be extended from the quarter plane $\mathfrak{R}^{+} \times \mathfrak{R}^{+}$to the whole plane $\Re \times \Re$ using the approach developed in [12]. The extended boundary integral operator will play a crucial role in our analysis. The second subsection contains $a$ priori estimates of norms of solutions of the linear problems and of norms of the boundary integral operators. 


\subsection{Solution formulas for linear problems}

Consider first the non-homogeneous problem

$$
\begin{aligned}
& u_{t}+\rho u_{x}-u_{x x}+u_{x x x}=0, \quad \text { for } x, t \geqslant 0, \\
& u(x, 0)=0, \quad u(0, t)=h(t),
\end{aligned}
$$

where $\rho \geqslant 0$ is a given constant.

Proposition 2.1. The solution of (2.1) may be written in the form

$$
u(x, t)=\left[W_{b d r}(t) h\right](x)=\left[U_{b}(t) h\right](x)+\overline{\left[U_{b}(t) h\right](x)}
$$

where, for $x, t \geqslant 0$,

$$
\left[U_{b}(t) h\right](x)=\frac{1}{2 \pi} \int_{\sqrt{\rho}}^{\infty} e^{i t\left(\mu^{3}-\rho \mu\right)} e^{(\alpha(\mu)+i \beta(\mu)) x}\left(3 \mu^{2}-\rho\right) \int_{0}^{\infty} e^{-i \xi\left(\mu^{3}-\rho \mu\right)} h(\xi) d \xi d \mu .
$$

Here, both $\alpha(\mu)$ and $\beta(\mu)$ are real-valued functions with $\alpha(\mu)<0$ for all $\mu \in \mathfrak{R}^{+}$and

$$
\alpha(\mu) \sim-\frac{\sqrt{3 \mu^{2}-4 \rho}}{2}, \quad \beta(\mu) \sim-\frac{\mu}{2}
$$

as $\mu \rightarrow+\infty$.

Proof. Let $\tilde{u}$ and $\tilde{h}$ denote the Laplace transform of $u$ and $h$ with respect to $t$, respectively. By applying the Laplace transform to both sides of the equation in (2.1), one obtains

$$
\lambda \tilde{u}(x, \lambda)+\rho \tilde{u}_{x}(x, \lambda)+\tilde{u}_{x x x}(x, \lambda)-\tilde{u}_{x x}(x, \lambda)=0, \quad \tilde{u}(0, \lambda)=\tilde{h}(\lambda) .
$$

As both $\tilde{u}(x, \lambda)$ and $\tilde{u}_{x}(x, \lambda)$ tend to zero as $x \rightarrow+\infty$, it is concluded that for any $\lambda$ with $\operatorname{Re} \lambda>0$,

$$
\tilde{u}(x, \lambda)=\tilde{h}(\lambda) e^{r_{1}(\lambda) x}
$$

where $r_{1}(\lambda)$ is the unique solution of

$$
\lambda+r^{3}+\rho r-r^{2}=0
$$

satisfying $\operatorname{Re} r_{1}(\lambda)<0$. Thus, for any fixed $\gamma>0$, one has the representation

$$
u(x, t)=\frac{1}{2 \pi i} \int_{-i \infty+\gamma}^{i \infty+\gamma} e^{\lambda t} \tilde{h}(\lambda) e^{r_{1}(\lambda) x} d \lambda .
$$

Using the fact that the right-hand side of this relation is continuous in $\gamma$ up to $\gamma=0$, there obtains the simpler looking formula

$$
u(x, t)=\frac{1}{2 \pi i} \int_{0}^{i \infty} e^{\lambda t} \tilde{h}(\lambda) e^{r_{1}(\lambda) x} d \lambda+\frac{1}{2 \pi i} \int_{-i \infty}^{0} e^{\lambda t} \tilde{h}(\lambda) e^{r_{1}(\lambda) x} d \lambda .
$$

For each $\lambda$ on the positive imaginary axis, write $\lambda$ in the form $\lambda=i\left(\mu^{3}-\rho \mu\right)$ for the unique value of $\mu$ in the interval $\sqrt{\rho} \leqslant \mu<+\infty$. In terms of $\mu$, the quantity $r_{1}(\lambda)$ has the form

$$
r_{1}(\lambda)=\alpha(\mu)+i \beta(\mu)
$$

with $\alpha(\mu)<0$ and

$$
\alpha(\mu) \sim-\frac{\sqrt{3 \mu^{2}-4 \rho}}{2}, \quad \beta(\mu) \sim-\frac{\mu}{2}
$$


as $\mu \rightarrow+\infty$. Recalling that

$$
\tilde{h}(\lambda)=\int_{0}^{\infty} e^{-\lambda t} h(t) d t
$$

a change of variables and straightforward calculation then reveals that for $x, t \geqslant 0$,

$$
\frac{1}{2 \pi i} \int_{0}^{i \infty} e^{\lambda t} \tilde{h}(\lambda) e^{r_{1}(\lambda) x} d \lambda=\left[U_{b}(t) h\right](x)
$$

and, similarly, that

$$
\frac{1}{2 \pi i} \int_{-i \infty}^{0} e^{\lambda t} \tilde{h}(\lambda) e^{r_{1}(\lambda) x} d \lambda=\overline{\left[U_{b}(t) h\right](x)}
$$

thus completing the proof.

Next, consider the same linear problem posed with zero boundary condition, but non-trivial initial data, viz.

$$
\left.\begin{array}{l}
u_{t}+\rho u_{x}-u_{x x}+u_{x x x}=0, \quad \text { for } x, t \geqslant 0, \\
u(x, 0)=\phi(x), \quad u(0, t)=0 .
\end{array}\right\}
$$

By semigroup theory, its solution may be obtained in the form

$$
u(t)=W_{c}(t) \phi,
$$

where the spatial variable is suppressed and $W_{c}(t)$ is the $C_{0}$-semigroup in the space $L^{2}\left(\Re^{+}\right)$generated by the operator

$$
A f=-f^{\prime \prime \prime}-\rho f^{\prime}+f^{\prime \prime}
$$

with the domain

$$
\mathcal{D}(A)=\left\{f \in H^{3}\left(\Re^{+}\right) \mid f(0)=0\right\} .
$$

Moreover, by Duhamel's formula, one may use the semigroup $W_{c}(t)$ to formally write the solution of the forced linear problem with zero auxiliary data,

$$
\begin{aligned}
& u_{t}+\rho u_{x}-u_{x x}+u_{x x x}=f, \quad \text { for } x, t \geqslant 0, \\
& u(x, 0)=0, \quad u(0, t)=0,
\end{aligned}
$$

in the form

$$
u(t)=\int_{0}^{t} W_{c}(t-\tau) f(\cdot, \tau) d \tau .
$$

Recall the explicit solution formula for the pure initial-value problem for the linear KdV-Burgers equation posed on the whole line $\Re$, viz.

$$
\left.\begin{array}{ll}
u_{t}+\rho u_{x}-u_{x x}+u_{x x x}=0, & x, t \in \Re, \\
u(x, 0)=\phi(x), & x \in \Re,
\end{array}\right\}
$$

namely

$$
u(x, t)=W_{\Re}(t) \phi(x)=\frac{1}{2 \pi} \int_{-\infty}^{\infty} e^{i\left(\xi^{3}-\rho \xi\right) t-\xi^{2} t} e^{i x \xi} \int_{-\infty}^{\infty} e^{-i y \xi} \phi(y) d y d \xi
$$


obtained by taking the Fourier transform in the spatial variable $x$. As the formula for $W_{\mathfrak{R}}(t)$ is explicit and simple, it is advantageous to give a representation of $W_{c}(t)$ in terms of $W_{\Re}(t)$ with a correction that involves $\mathcal{W}_{b d r}(t)$. Because of the Burgers-type term, notice that if $\phi \in H^{s}(\Re)$ for any $s \in \Re$, then $u(x, t)$ is an $H^{\infty}(\Re)$-function of $x$ for any $t>0$, and so is $C^{\infty}$ in the domain $\{(x, t): x \in \Re, t>0\}$. Hence, the trace of $u$ at $x=0$, say, is a well-defined function.

Let a function $\phi$ be defined on the half line $\Re^{+}$and let $\phi^{*}$ be an extension of $\phi$ to the whole line $\Re$. The mapping $\phi \mapsto \phi^{*}$ can be organized so that it defines a bounded linear operator $B$ from $H^{s}\left(\Re^{+}\right)$to $H^{s}(\Re)$. Henceforth, $\phi^{*}=B \phi$ will refer to the result of such an extension operator applied to $\phi \in H^{s}\left(\Re^{+}\right)$. Assume that $v=v(x, t)$ is the solution of

$$
v_{t}+\rho v_{x}-v_{x x}+v_{x x x}=0, \quad v(x, 0)=\phi^{*}(x),
$$

for $x \in \Re, t \geqslant 0$. If $g(t)=v(0, t)$, then $v_{g}=v_{g}(x, t)=\mathcal{W}_{b d r}(t) g$ is the corresponding solution of the nonhomogeneous boundary-value problem (2.1) with boundary condition $h(t)=g(t)$ for $t \geqslant 0$. It is clear that for $x>0$, the function $v(x, t)-v_{g}(x, t)$ solves the IBVP (2.4), and this leads directly to a representation of the semigroup $W_{c}(t)$ in terms of $\mathcal{W}_{b d r}(t)$ and $W_{\Re}(t)$.

Proposition 2.2. For a given $s$ and $\phi \in H^{s}\left(\Re^{+}\right)$with $\phi(0)=0$, if $s>\frac{1}{2}$, if $\phi^{*}$ is its extension to $\Re$ as described above, then $W_{c}(t) \phi$ may be written in the form

$$
W_{c}(t) \phi=W_{\Re}(t) \phi^{*}-\mathcal{W}_{b d r}(t) g
$$

for any $x, t>0$, where $g$ is the trace of $W_{\Re}(t) \phi^{*}$ at $x=0$.

In a similar manner, one may derive an alternative representation for solutions of the inhomogeneous IBVP (2.6).

Proposition 2.3. If $f^{*}(\cdot, t)=B f(\cdot, t)$ is an extension of $f$ from $\mathfrak{R}^{+} \times \mathfrak{R}^{+}$to $\Re \times \mathfrak{R}^{+}$, say, then the solution $u$ of (2.6) may be written in the form

$$
u(\cdot, t)=\int_{0}^{t} W_{\Re}(t-\tau) f^{*}(\cdot, \tau) d \tau-\mathcal{W}_{b d r}(t) v
$$

for any $x, t \geqslant 0$ where $v \equiv v(t)$ is the trace of $\int_{0}^{t} W_{\Re}(t-\tau) f^{*}((\cdot, \tau) d \tau$ at $x=0$.

The solution formulas in Propositions 2.2 and 2.3 hold only for $x>0$ and $t>0$. It will be convenient to extend them in such a way that they hold for all $x, t \in \Re$. This will provide a context in which to establish the well-posedness of the non-linear problem in the framework of Bourgain spaces. Note that the term $W_{\Re}(t)$ can be redefined to be

$$
W_{\Re}(t) \phi=\frac{1}{2 \pi} \int_{-\infty}^{\infty} e^{i\left(\xi^{3}-\rho \xi\right) t-\xi^{2}|t|} e^{i x \xi} \int_{-\infty}^{\infty} e^{-i y \xi} \phi(y) d y d \xi
$$

for all $x, t \in \Re$ without disturbing the validity of (2.10) in $\Re^{+} \times \Re^{+}$. Thus it is only necessary to extend the second term in both formulas from $\mathfrak{R}^{+} \times \mathfrak{R}^{+}$to $\Re \times \Re$ to effect an extension of $W_{c}$.

Presented now are two different types of extensions of the boundary integral operator $\mathcal{W}_{b d r}(t)$. To begin, rewrite $\mathcal{W}_{b d r}(t)$ in the form

$$
\begin{aligned}
{\left[\mathcal{W}_{b d r}(t) h\right](x)=} & \frac{1}{2 \pi} \operatorname{Re} \int_{\sqrt{\rho}}^{\infty} e^{i t\left(\mu^{3}-\rho \mu\right)} e^{(\alpha(\mu)+i \beta(\mu)) x}\left(3 \mu^{2}-\rho\right) \int_{0}^{\infty} e^{-i\left(\mu^{3}-\rho \mu\right) \xi} h(\xi) d \xi d \mu \\
= & \frac{1}{2 \pi} \operatorname{Re} \int_{\sqrt{\rho}}^{4 \sqrt{\rho}} e^{i \mu^{3} t-i \rho \mu t} e^{(\alpha(\mu)+i \beta(\mu)) \phi_{3}(x)}\left(3 \mu^{2}-\rho\right) \phi_{1}(\mu) \int_{0}^{\infty} e^{-i\left(\mu^{3}-\rho \mu\right) \xi} h(\xi) d \xi d \mu \\
& +\frac{1}{2 \pi} \operatorname{Re} \int_{\frac{2 \sqrt{ }}{\sqrt{3}}}^{\infty} e^{i \mu^{3} t-i \rho \mu t} e^{(\alpha(\mu)+i \beta(\mu)) x}\left(3 \mu^{2}-\rho\right) \phi_{2}(\mu) \int_{0}^{\infty} e^{-i\left(\mu^{3}-\rho \mu\right) \xi} h(\xi) d \xi d \mu \\
:= & \frac{1}{2 \pi}\left\{I_{1}(x, t)+I_{2}(x, t)\right\}
\end{aligned}
$$


where $\phi_{1}(\mu)$ and $\phi_{2}(\mu)$ are non-negative cut-off functions satisfying

$$
\phi_{1}(\mu)+\phi_{2}(\mu)=1 \quad \text { for all } \mu \in \mathfrak{R}^{+}
$$

with supp $\phi_{1} \subset(-1,4 \sqrt{\rho})$, supp $\phi_{2} \subset(3 \sqrt{\rho}, \infty)$ and $\phi_{3}(x)$ is a smooth function on $\Re$ such that

$$
\phi_{3}(x)= \begin{cases}x & \text { for } x \geqslant 0 \\ 0 & \text { for } x \leqslant-1\end{cases}
$$

The integral $I_{1}(x, t)$ is naturally defined for all values of $x$ and $t$ and, viewed as a function defined on $\Re \times \Re$, is in fact $C^{\infty}$-smooth there, with all its derivatives decreasing rapidly as $x \rightarrow \pm \infty$. Thus, no complicated extension of $I_{1}$ is required as the obvious one suffices. It is otherwise for $I_{2}$. To discuss $I_{2}(x, t)$, it is convenient to let $\mu(\lambda)$ denote the positive solution of the cubic equation

$$
\mu^{3}-\rho \mu=\lambda
$$

for $\lambda \geqslant 0$ and $\mu \geqslant \sqrt{\rho}$, while $\mu(\lambda)=-\mu(-\lambda)$ for $\lambda<0$. By a change of variables, the integral $I_{2}$ can be rewritten in the form

$$
\begin{aligned}
I_{2}(x, t) & =\operatorname{Re} \int_{\frac{2 \sqrt{\rho}}{3 \sqrt{3}}}^{\infty} \int_{0}^{\infty} e^{i \lambda t} e^{\left(\alpha_{\mu}(\lambda)+i \beta_{\mu}(\lambda)\right) x} e^{-i \lambda s} \phi_{2}(\mu(\lambda)) h(s) d s d \lambda \\
& =\int_{\frac{2 \sqrt{\rho}}{3 \sqrt{3}}}^{\infty} \int_{0}^{\infty} e^{\alpha_{\mu}(\lambda) x} \cos \left(\lambda(t-s)+\beta_{\mu}(\lambda) x\right) \phi_{2}(\mu(\lambda)) h(s) d s d \lambda \\
& :=E(x, t)
\end{aligned}
$$

for $x \geqslant 0$ with $\alpha_{\mu}(\lambda):=\alpha(\mu(\lambda))$ and $\beta_{\mu}(\lambda):=\beta(\mu(\lambda))$. Denote the extension of $E(x, t)$ to $x<0$ by $g(x, t)$ so that

$$
I_{2}(x, t)= \begin{cases}E(x, t), & x \geqslant 0 \\ g(x, t), & x<0\end{cases}
$$

where $g(x, t)$ is to be defined. As in [12], the Fourier transform of $I_{2}$ may be decomposed as follows:

$$
\begin{aligned}
\mathcal{F}_{x, t}\left[I_{2}\right]= & \mathcal{F}_{t}\left[\int_{0}^{\infty}(E(x, t) \cos (x \xi)+g(-x, t) \cos (x \xi)) d x\right] \\
& +\frac{i}{\pi} \int_{-\infty}^{\infty} \frac{1}{\xi-\eta} \mathcal{F}_{t}\left[\int_{0}^{\infty} \cos (\eta x) E(x, t) d x-\int_{0}^{\infty} \cos (\eta x) g(-x, t) d x\right] d \eta .
\end{aligned}
$$

Note that different choices of $g(x, t)$ give different extensions of $E(x, t)$ to $x<0$. The following two choices of $g(x, t)$ will be used in this article.

(i) For $x>0$, choose $g(-x, t)$ such that

$$
\begin{aligned}
\mathcal{F}_{t}\left[\int_{0}^{\infty} g(-x, t) \cos (x \xi) d x\right](\tau)= & -\mathcal{F}_{t}\left[\int_{0}^{\infty} E(x, t) \cos (x \xi)\right](\tau) \Theta(\xi, \tau) \\
& +\mathcal{F}_{t}\left[\int_{0}^{\infty} E(x, t) \cos (x \xi) d x\right](\tau)(1-\Theta(\xi, \tau)) v(\xi) \omega(\tau)
\end{aligned}
$$

where $\Theta(\xi, \tau)=\chi\left(|\xi|-\delta|\tau|^{1 / 3}\right)$ with $\delta>0$ fixed, $0 \leqslant \chi(\xi) \leqslant 1$ everywhere,

$$
\chi(\xi)= \begin{cases}1, & \xi<0, \\ 0, & \xi>0,\end{cases}
$$


whilst

$$
v(\xi)= \begin{cases}1 & \text { if }|\xi| \geqslant 1, \\ 0 & \text { if }|\xi|<1,\end{cases}
$$

and $\omega(\tau)$ is a smooth and bounded function to be specified momentarily. It is easy to see that such a $g$ is a combination of even and odd extensions, viz.

$$
\mathcal{F}_{x, t}\left[I_{2}\right]:=\hat{I}_{21}(\xi, \tau)+\hat{I}_{22}(\xi, \tau)
$$

where

$$
\hat{I}_{21}(\xi, \tau)=\mathcal{F}_{t}\left[\int_{0}^{\infty} E(x, t) \cos (x \xi) d x\right](\tau)(1-\Theta(\xi, \tau))(1+\nu(\xi) \omega(\tau))
$$

and

$$
\begin{aligned}
\hat{I}_{22}(\xi, \tau)= & \frac{i}{\pi} \int_{-\infty}^{\infty} \frac{1}{\xi-\eta} \mathcal{F}_{t}\left[\int_{0}^{\infty} E(x, t) \cos (x \eta) d x\right](\tau)(2 \Theta(\eta, \tau)+(1-\Theta(\eta, \tau))(1+v(\xi) \omega(\tau))) d \eta \\
= & \frac{i}{\pi} \int_{0}^{\infty}\left(\frac{1}{\xi-\eta}+\frac{1}{\xi+\eta}\right) \mathcal{F}_{t}\left[\int_{0}^{\infty} E(x, t) \cos (x \eta) d x\right](\tau) \\
& \times(2 \Theta(\eta, \tau)+(1-\Theta(\eta, \tau))(1+v(\xi) \omega(\tau))) d \eta .
\end{aligned}
$$

Because of the algebraic identity

$$
\frac{1}{\xi-\eta}+\frac{1}{\xi+\eta}=\frac{2}{\xi}\left(1+\frac{\eta^{2}}{\xi^{2}-\eta^{2}}\right)
$$

we may write $\hat{I}_{22}(\xi, \tau)$ as

$$
\begin{aligned}
\hat{I}_{22}(\xi, \tau)= & \frac{2 i}{\pi \xi} \int_{0}^{\infty} \mathcal{F}_{t}\left[\int_{0}^{\infty} E(x, t) \cos (x \eta) d x\right](\tau)(2 \Theta(\eta, \tau)+(1-\Theta(\eta, \tau))(1+v(\xi) \omega(\tau))) d \eta \\
& +\frac{2 i}{\pi \xi} \int_{0}^{\infty} \frac{(\eta / \xi)^{2}}{1-(\eta / \xi)^{2}} \mathcal{F}_{t}\left[\int_{0}^{\infty} E(x, t) \cos (x \eta) d x\right](\tau) \\
& \times(2 \Theta(\eta, \tau)+(1-\Theta(\eta, \tau))(1+v(\xi) \omega(\tau))) d \eta \\
:= & Q_{1}(\xi, \tau)+Q_{2}(\xi, \tau) .
\end{aligned}
$$

Here, the Fourier transform $\mathcal{F}_{t}\left[\int_{0}^{\infty} E(x, t) \cos (x \eta) d x\right]$ may be expressed in the form

$$
\mathcal{F}_{t}\left[\int_{0}^{\infty} E(x, t) \cos (x \eta) d x\right](\tau)=\sum_{m=1}^{4} K_{m 1}(\eta, \tau) \phi_{2}(\mu(\tau)) \hat{h}(\tau)+\sum_{m=1}^{4} K_{m 2}(\eta,-\tau) \phi_{2}(\mu(-\tau)) \hat{h}(-\tau)
$$

where

$$
\begin{aligned}
& \hat{h}(\tau)=\int_{0}^{\infty} e^{-i \tau s} h(s) d s, \\
& K_{11}(\eta, \lambda)=\frac{-\alpha_{\mu}(\lambda)}{2\left(\alpha_{\mu}^{2}(\lambda)+\left(\eta+\beta_{\mu}(\lambda)\right)^{2}\right)}, \\
& K_{21}(\eta, \lambda)=\frac{-\alpha_{\mu}(\lambda)}{2\left(\alpha_{\mu}^{2}(\lambda)+\left(\eta-\beta_{\mu}(\lambda)^{2}\right)\right.},
\end{aligned}
$$




$$
\begin{aligned}
& K_{31}(\eta, \lambda)=\frac{\alpha_{\mu}^{2}(\lambda) \beta_{\mu}(\lambda) i}{\left(\alpha_{\mu}^{2}(\lambda)+\left(\eta+\beta_{\mu}(\lambda)\right)^{2}\right)\left(\alpha_{\mu}^{2}(\lambda)+\left(\eta-\beta_{\mu}(\lambda)\right)^{2}\right)}, \\
& K_{41}(\eta, \lambda)=\frac{\left(\beta_{\mu}^{2}(\lambda)-\eta^{2}\right) \beta_{\mu}(\lambda) i}{\left(\alpha_{\mu}^{2}(\lambda)+\left(\eta+\beta_{\mu}(\lambda)\right)^{2}\right)\left(\alpha_{\mu}^{2}(\lambda)+\left(\eta-\beta_{\mu}(\lambda)\right)^{2}\right)}
\end{aligned}
$$

and

$$
\begin{cases}K_{12}(\eta, \lambda)=K_{11}(\eta, \lambda), & K_{22}(\eta, \lambda)=K_{21}(\eta, \lambda) \\ K_{32}(\eta, \lambda)=-K_{31}(\eta, \lambda), & K_{42}(\eta, \lambda)=-K_{41}(\eta, \lambda) .\end{cases}
$$

A straightforward calculation reveals that

$$
\left|\int_{0}^{\infty} \sum_{m=1}^{4} K_{m 1}(\eta, \tau)(1-\Theta(\eta, \tau)) d \eta\right| \geqslant C_{2}>0,
$$

for a fixed constant $C_{2}$ independent of $\tau$, and so one can choose a $C^{\infty}$ smooth function $\omega(\tau)$ (see [12]) such that for all $\tau$,

$$
Q_{1}(\xi, \tau) \equiv 0, \quad \text { for all }|\xi| \geqslant 1 .
$$

Hence, for $|\xi|>1$,

$$
\hat{I}_{22}(\xi, \tau)=Q_{2}(\xi, \tau) \text {. }
$$

Thus, when $|\xi|>1$ and $\tau \geqslant 0$,

$$
\hat{I}_{22}(\xi, \tau)=\frac{2 i C_{2}}{\xi} \int_{0}^{\infty} \frac{\eta^{2}}{\xi^{2}-\eta^{2}}\left[\sum_{m=1}^{4} K_{m 1}(\eta, \tau) \phi_{2}(\mu(\tau)) \hat{h}(\tau)\right](2 \Theta(\eta, \tau)+(1-\Theta(\eta, \tau))(1+\omega(\tau))) d \eta,
$$

whereas

$$
\hat{I}_{22}(\xi, \tau)=\frac{2 i C_{2}}{\xi} \int_{0}^{\infty} \frac{\eta^{2}}{\xi^{2}-\eta^{2}}\left[\sum_{m=1}^{4} K_{m 1}(\eta,-\tau) \phi_{2}(\mu(-\tau)) \hat{h}(\tau)\right](2 \Theta(\eta, \tau)+(1-\Theta(\eta, \tau))(1+\omega(\tau))) d \eta
$$

when $|\xi| \geqslant 1$ and $\tau<0$. The boundary integral operator corresponding to this extension of $\mathcal{W}_{b d r}(t)$ is denoted by $\mathcal{B I}_{1}(t)$.

(ii) Choose $g(-x, t)$ such that

$$
\begin{aligned}
\mathcal{F}_{t}\left[\int_{0}^{\infty} g(-x, t) \cos (x \xi) d x\right](\tau)= & -\mathcal{F}_{t}\left[\int_{0}^{\infty} E(x, t) \cos (x \xi)\right](\tau)(1-\Theta(\xi, \tau)) \\
& +\mathcal{F}_{t}\left[\int_{0}^{\infty} E(x, t) \cos (x \xi) d x\right](\tau) \Theta(\xi, \tau) v(\xi) \omega(\tau)
\end{aligned}
$$

where $\Theta$ is as before. In this case, we have

$$
\mathcal{F}_{x, t}\left[I_{2}\right]:=\hat{I}_{21}^{*}(\xi, \tau)+\hat{I}_{22}^{*}(\xi, \tau)
$$

with

$$
\hat{I}_{21}^{*}(\xi, \tau)=\mathcal{F}_{t}\left[\int_{0}^{\infty} E(x, t) \cos (x \xi) d x\right] \Theta(\xi, \tau)(1+v(\xi) \omega(\tau))
$$

and 


$$
\begin{aligned}
\hat{I}_{22}^{*}(\xi, \tau)= & \frac{i}{\pi} \int_{-\infty}^{\infty} \frac{1}{\xi-\eta} \mathcal{F}_{t}\left[\int_{0}^{\infty} E(x, t) \cos (x \eta) d x\right](2(1-\Theta(\eta, \tau))+\Theta(\eta, \tau)(1+v(\xi) \omega(\tau))) d \eta \\
= & \frac{i}{\pi} \int_{0}^{\infty}\left(\frac{1}{\xi-\eta}+\frac{1}{\xi+\eta}\right) \mathcal{F}_{t}\left[\int_{0}^{\infty} E(x, t) \cos (x \eta) d x\right] \\
& \times(2(1-\Theta(\eta, \tau))+\Theta(\eta, \tau)(1+v(\xi) \omega(\tau))) d \eta \\
= & \frac{2 i C_{2}}{\xi} \int_{0}^{\infty} \mathcal{F}_{t}\left[\int_{0}^{\infty} E(x, t) \cos (x \eta) d x\right](2(1-\Theta(\eta, \tau))+\Theta(\eta, \tau)(1+v(\xi) \omega(\tau))) d \eta \\
& +\frac{2 i C_{2}}{\xi} \int_{0}^{\infty} \frac{(\eta / \xi)^{2}}{1-(\eta / \xi)^{2}} \mathcal{F}_{t}\left[\int_{0}^{\infty} E(x, t) \cos (x \eta) d x\right](2(1-\Theta(\eta, \tau))+\Theta(\eta, \tau)(1+v(\xi) \omega(\tau))) d \eta \\
:= & Q_{1}^{*}(\xi, \tau)+Q_{2}^{*}(\xi, \tau) .
\end{aligned}
$$

As in case (i), one can choose an appropriate $\omega(\tau)$ so that

$$
Q_{1}^{*}(\xi, \tau)=0 \quad \text { for }|\xi|>1 \text { and any } \tau \in \Re .
$$

The boundary integral operator corresponding to this extension of $\mathcal{W}_{b d r}(t)$ is denoted by $\mathcal{B I}_{2}(t)$.

\subsection{Linear estimates}

In this subsection, estimates are provided for solutions of the associated linear version of the KdV-Burgers equation. These are needed in establishing the well-posedness of the non-linear problems in the next section.

For given $s \in \Re, 0 \leqslant b \leqslant 1$ and any measurable function $w \equiv w(x, t): \Re \times \Re \rightarrow \Re$, define

$$
\Lambda_{s, b}(w)=\left(\int_{-\infty}^{\infty} \int_{-\infty}^{\infty}\left\langle i\left(\tau-\left(\xi^{3}-\rho \xi\right)\right)+\xi^{2}\right\rangle^{2 b}\langle\xi\rangle^{2 s}|\hat{w}(\xi, \tau)|^{2} d \xi d \tau\right)^{1 / 2}
$$

where $\langle\cdot\rangle=\left(1+|\cdot|^{2}\right)^{1 / 2}$. Let $X_{s, b}$ be the completion of the space of all functions $w$ satisfying

$$
\|w\|_{X_{s, b}}:=\Lambda_{s, b}(w)<\infty
$$

and let

$$
\mathcal{X}_{s, b} \equiv C\left(\Re ; H^{s}(\Re)\right) \cap X_{s, b}
$$

with the norm

$$
\|w\|_{\mathcal{X}_{s, b}}=\left(\sup _{t \in \mathfrak{R}}\|w(\cdot, t)\|_{H^{s}(\mathfrak{R})}^{2}+\|w\|_{X_{s, b}}^{2}\right)^{1 / 2} .
$$

First, consider the semigroup $\left\{W_{\Re}(t)\right\}_{t} \geqslant 0$ associated to the linear KdV-Burgers equation posed on the whole line $\Re$. Recall that for any $\phi \in \mathcal{S}^{\prime}$, the space of tempered distributions, and $t \geqslant 0$,

$$
\mathcal{F}_{x}\left(W_{\Re}(t) \phi\right)(\xi)=\exp \left[-\xi^{2} t+i\left(\xi^{3}-\rho \xi\right) t\right] \hat{\phi}(\xi) .
$$

Extend $W_{\Re}$ to a linear operator defined on the whole real axis by setting

$$
\mathcal{F}_{x}\left(W_{\Re}(t) \phi\right)(\xi)=\exp \left[-\xi^{2}|t|+i\left(\xi^{3}-\rho \xi\right) t\right] \hat{\phi}(\xi)
$$

for all $t \in \Re$. The proof of the following proposition regarding $\left\{W_{\Re}(t)\right\}_{0}^{\infty}$ is a minor modification of arguments found in [47]. 
Proposition 2.4. Let $s \in \mathfrak{R}, 0<b \leqslant 1$ and $0<\delta<\frac{1}{2}$ be given. Let $\psi$ be a $C^{\infty}$ smooth function with compact support.

(i) There exists a constant $C$ depending only on $s, b$ and $\psi$ such that

$$
\left\|\psi(t) W_{\Re}(t) \phi\right\|_{\mathcal{X}_{s, b}} \leqslant C\|\phi\|_{H^{s}(\Re)} .
$$

(ii) There exists $C_{\delta}$ such that for all $u \in X_{s,-\frac{1}{2}+\delta}$,

$$
\left\|\psi(t) \int_{0}^{t} W_{\Re(}\left(t-t^{\prime}\right) f\left(t^{\prime}\right) d t^{\prime}\right\|_{\mathcal{X}_{s, \frac{1}{2}}} \leqslant C_{\delta}\|f\|_{X_{s,-\frac{1}{2}+\delta}} .
$$

(iii) There exists a constant $C$ depending only on s such that

$$
\sup _{x \in \Re}\left\|W_{\Re}(t) \phi\right\|_{H_{t}^{\frac{s}{3}}(\Re)} \leqslant C\|\phi\|_{H^{s}(\Re)} .
$$

(iv) For all $f \in X_{s,-\frac{1}{2}+\delta}$, the correspondence

$$
t \mapsto \int_{0}^{t} W_{\Re}\left(t-t^{\prime}\right) f\left(t^{\prime}\right) d t^{\prime}
$$

lies in $C\left(\Re^{+}, H^{s+2 \delta}(\Re)\right)$. In addition, if $\left\{f_{n}\right\}$ is a sequence with $f_{n} \rightarrow 0$ in $X_{s,-\frac{1}{2}+\delta}$, then

$$
\left\|\int_{0}^{t} W_{\Re}\left(t-t^{\prime}\right) f_{n}\left(t^{\prime}\right) d t^{\prime}\right\|_{L^{\infty}\left(\Re^{+}, H^{s+2 \delta}(\Re)\right)} \rightarrow 0 \quad \text { as } n \rightarrow \infty .
$$

Next, attention is turned to the spatial traces of $W_{\Re}(t) \phi$ and $\int_{0}^{t} W_{\Re}\left(t-t^{\prime}\right) f\left(\cdot, t^{\prime}\right) d t$.

Proposition 2.5. Let $s \in[-1,5]$ be given. There exists a constant $C$ depending only on such that

$$
\sup _{x \in \Re}\left\|W_{\Re}(t) \phi\right\|_{H_{t}^{\frac{s+1}{3}}(\Re)} \leqslant C\|\phi\|_{H^{s}(\Re)}
$$

and

$$
\sup _{x \in \Re}\left\|\partial_{x} W_{\Re}(t) \phi\right\|_{H_{t}^{\frac{s}{3}}(\Re)} \leqslant C\|\phi\|_{H^{s}(\Re)}
$$

for any $\phi \in H^{s}(\Re)$.

The proof of this proposition is based on the following technical lemma which plays the same role in our arguments as would a Plancherel Theorem. The proof of the lemma is postponed and provided in Appendix B.

Lemma 2.6. Suppose $0<v<1$ and let I be the integral operator defined by

$$
[I(f)](t):=\int_{-\infty}^{\infty} e^{i t \eta+\mu(\eta)|t|} f(\eta) d \eta, \quad \text { for } t \in \Re,
$$

where $\mu: \Re \rightarrow(-\infty, 0]$ is a continuous function satisfying

$$
|\mu(\eta)| \sim \eta^{\nu} \quad \text { as } \eta \rightarrow 0 \text { and }|\mu(\eta)| \sim|\eta|^{\frac{1}{3}} \quad \text { as }|\eta| \rightarrow \infty .
$$

Then, $I$ is a bounded linear operator on $L^{2}(\Re)$, which is to say, there exists a constant $C$ such that

$$
\|I(f)\|_{L^{2}(\Re)} \leqslant C\|f\|_{L^{2}(\Re)}
$$

for all $f \in L^{2}(\Re)$. 
Proof of Proposition 2.5. We only provide the proof of (2.18). The proof of (2.19) is very similar and is therefore omitted. Let $u(x, t)=W_{\Re}(t) \phi$. Then, a change of variables reveals that

$$
\begin{aligned}
u(x, t) & =\int_{-\infty}^{\infty} e^{i x \xi} e^{\left[i\left(\xi^{3}-\rho \xi\right)\right] t-\xi^{2}|t|} \hat{\phi}(\xi) d \xi \\
& =\int_{|\lambda| \geqslant \sqrt{\rho}} e^{i x v(\lambda)} e^{i \lambda t-\nu^{2}(\lambda)|t|}\left(3 v^{2}(\lambda)-\rho\right)^{-1} \hat{\phi}(v(\lambda)) d \lambda,
\end{aligned}
$$

where $\xi=v(\lambda)$ solves the equation

$$
\xi^{3}-\rho \xi=\lambda
$$

with $v(\lambda) \sim \lambda^{\frac{1}{3}}$ as $\lambda \rightarrow \infty$. By Lemma 2.6, the inequality

$$
\|u(x, \cdot)\|_{L^{2}(\Re)} \leqslant C\left(\int_{-\infty}^{\infty}\left|\left(3 v^{2}(\lambda)-\rho\right)^{-1} \hat{\phi}(v(\lambda))\right|^{2} d \lambda\right)^{\frac{1}{2}} \leqslant C\|\phi\|_{H^{-1}(\Re)}
$$

holds for any $x \in \Re$. Note that

$$
u_{t}(x, t)=\int_{-\infty}^{\infty} e^{i x \xi} e^{\left[i\left(\xi^{3}-\rho \xi\right)\right] t-\xi^{2}|t|}\left[i\left(\xi^{3}-\rho \xi\right)+\kappa(t) \xi^{2}\right] \hat{\phi}(\xi) d \xi,
$$

where $\kappa(t)=-1$ if $t \geqslant 0$ and $\kappa(t)=1$ if $t<0$. Applying Lemma 2.6 again yields that

$$
\|u(x, \cdot)\|_{H^{1}(\Re)} \leqslant C\|\phi\|_{H^{2}(\Re)}
$$

for any $x \in \Re$. Similarly, one can show that

$$
\|u(x, \cdot)\|_{H^{2}(\Re)} \leqslant C\|\phi\|_{H^{5}(\Re)}
$$

for any $x \in \mathfrak{R}$. The inequality (2.18) then follows by interpolation. The proof is thereby completed.

Proposition 2.7. Let $0 \leqslant b<1 / 2,-1 \leqslant s \leqslant 2-3 b, \psi \in C_{0}^{\infty}(\Re)$ and

$$
w(x, t)=\int_{0}^{t} W_{\Re}\left(t-t^{\prime}\right) f\left(\cdot, t^{\prime}\right) d t^{\prime} .
$$

Then, there exists $C$ depending only on $b$, s and $\psi$ such that

$$
\sup _{x \in \mathfrak{R}}\|\psi(\cdot) w(x, \cdot)\|_{H_{t}^{\frac{s+1}{3}}(\Re)} \leqslant C\|f\|_{X_{s,-b}}
$$

and

$$
\sup _{x \in \Re}\left\|\psi(t) w_{x}(x, t)\right\|_{H_{t}^{s / 3}(\Re)} \leqslant C\|f\|_{X_{s,-b}} .
$$

Proof. The proof presented below is based on the approach developed in [15,43], appropriately modified. As the proof of (2.21) is similar to that of (2.20), only (2.20) is proved.

First, observe that

$$
\begin{aligned}
& \psi(t) \int_{0}^{t} W_{\Re}\left(t-t^{\prime}\right) f\left(t^{\prime}\right) d t^{\prime} \\
& \quad=\psi(t) \int_{-\infty}^{\infty} \int_{-\infty}^{\infty} e^{i x \xi} \hat{f}(\xi, \tau) \psi\left(\left|\tau-\left(\xi^{3}-\rho \xi\right)\right|+\xi^{2}\right) \frac{e^{i \tau t}-e^{i t\left(\xi^{3}-\rho \xi\right)-\xi^{2} t}}{i\left[\tau-\left(\xi^{3}-\rho \xi\right)\right]+\xi^{2}} d \xi d \tau
\end{aligned}
$$




$$
\begin{aligned}
& \quad+\psi(t) \int_{-\infty}^{\infty} \int_{-\infty}^{\infty} e^{i x \xi} \hat{f}(\xi, \tau)\left(1-\psi\left(\left|\tau-\left(\xi^{3}-\rho \xi\right)\right|+\xi^{2}\right)\right) \frac{e^{i \tau t}-e^{i t\left(\xi^{3}-\rho \xi\right)-\xi^{2} t}}{i\left[\tau-\left(\xi^{3}-\rho \xi\right)\right]+\xi^{2}} d \xi d \tau \\
& :=A+B .
\end{aligned}
$$

By a Taylor series expansion, we see that

$$
A=\sum_{k=1}^{\infty} \frac{1}{k !} \psi_{k}(t) W_{\Re}(t) g
$$

with

$$
\hat{g}(\xi)=\int_{-\infty}^{\infty} \hat{f}(\xi, \tau)\left(i\left(\tau-\left(\xi^{3}-\rho \xi\right)\right)+\xi^{2}\right)^{k-1} \psi\left(\left|\tau-\left(\xi^{3}-\rho \xi\right)\right|+\xi^{2}\right) d \tau
$$

and

$$
\psi_{k}(t):=t^{k} \psi(t), \quad k=1,2, \ldots
$$

Note that

$$
\left\|\psi_{k}\right\|_{H^{\frac{s+1}{3}(\Re)}} \leqslant \int_{-\infty}^{\infty}\left|\hat{\psi}_{k}(\tau)\right|^{2}(1+|\tau|)^{2 \frac{s+1}{3}} d \tau \leqslant C(k+1)^{2}
$$

and

$$
\begin{aligned}
\|g\|_{H^{s}(\Re)}^{2} & \leqslant C \int_{-\infty}^{\infty}\langle\xi\rangle^{2 s}\left|\int_{-\infty}^{\infty} \hat{f}(\xi, \tau)\left(i\left(\tau-\left(\xi^{3}-\rho \xi\right)\right)+\xi^{2}\right)^{k-1} \psi\left(\left|\tau-\left(\xi^{3}-\rho \xi\right)\right|+\xi^{2}\right) d \tau\right|^{2} d \xi \\
& \leqslant C \int_{-\infty}^{\infty}\langle\xi\rangle^{2 s}\left|\int_{\left|\tau-\left(\xi^{3}-\rho \xi\right)\right|+\xi^{2}<1} \hat{f}(\xi, \tau) d \tau\right|^{2} d \xi \\
& \leqslant C \int_{-\infty}^{\infty}\langle\xi\rangle^{2 s}\left|\int_{-\infty}^{\infty} \frac{\hat{f}(\xi, \tau)}{1+\left|\tau-\left(\xi^{3}-\rho \xi\right)\right|+\xi^{2}} d \tau\right|^{2} d \xi
\end{aligned}
$$

because of the restriction on the support of $\psi$. Thus, applying (2.20) gives

$$
\begin{aligned}
\sup _{x \in \mathfrak{R}}\|A(x, \cdot)\|_{H_{t}^{\frac{s+1}{3}}(\Re)} & \leqslant C \sum_{k=1}^{\infty} \frac{(k+1)^{2}}{k !}\|g\|_{H^{s}(\Re)} \\
& \leqslant C\left(\int_{-\infty}^{\infty}\langle\xi\rangle^{2 s}\left|\int_{-\infty}^{\infty} \frac{\hat{f}(\xi, \tau)}{1+\left|\tau-\left(\xi^{3}-\rho \xi\right)\right|+\xi^{2}} d \tau\right|^{2} d \xi\right)^{\frac{1}{2}} \\
& \leqslant C\|f\|_{X_{s,-b}}
\end{aligned}
$$

because $0 \leqslant b<\frac{1}{2}$.

Next, consider the term $B$ which can be written

$$
B=B_{1}+B_{2}
$$

with

$$
B_{1}=-\psi(t) \int_{-\infty}^{\infty} e^{i\left(x \xi+t\left(\xi^{3}-\rho \xi\right)\right)-\xi^{2} t}\left(\int_{-\infty}^{\infty} \frac{1-\psi\left(\left|\tau-\left(\xi^{3}-\rho \xi\right)\right|+\xi^{2}\right)}{i\left(\tau-\left(\xi^{3}-\rho \xi\right)\right)+\xi^{2}} \hat{f}(\xi, \tau)\right) d \xi
$$


and

$$
B_{2}=\psi(t) \int_{-\infty}^{\infty} e^{i(x \xi+t \tau)-\xi^{2} t}\left(\int_{-\infty}^{\infty} \frac{1-\psi\left(\left|\tau-\left(\xi^{3}-\rho \xi\right)\right|+\xi^{2}\right)}{i\left(\tau-\left(\xi^{3}-\rho \xi\right)\right)+\xi^{2}} \hat{f}(\xi, \tau)\right) d \xi
$$

For $B_{1}$, Proposition 2.6 yields

$$
\sup _{x \in R}\left\|B_{1}(x, t)\right\|_{H_{t}^{\frac{s+1}{2}}(R)}^{2} \leqslant C \int_{-\infty}^{\infty}\langle\xi\rangle^{2 s}\left(\int_{-\infty}^{\infty} \frac{|\hat{f}(\xi, \tau)|}{1+\left|\tau-\left(\xi^{3}-\rho \xi\right)\right|+\xi^{2}} d \tau\right)^{2} d \xi \leqslant C\|f\|_{X_{s,-b}}^{2} .
$$

As for $B_{2}(x, t)$, note that

$$
\begin{aligned}
\sup _{x \in R}\left\|B_{2}(x, t)\right\|_{H_{t}^{\frac{s+1}{3}}(\Re)}^{2} & \leqslant C \int_{-\infty}^{\infty}(1+|\tau|)^{\frac{2(s+1)}{3}}\left(\int_{-\infty}^{\infty} \frac{\left|1-\psi\left(\left|\tau-\left(\xi^{3}-\rho \xi\right)\right|+\xi^{2}\right)\right|}{\left|\tau-\left(\xi^{3}-\rho \xi\right)\right|+\xi^{2}} \hat{f}(\xi, \tau) d \xi\right)^{2} d \tau \\
& \leqslant C \int_{-\infty}^{\infty}(1+|\tau|)^{\frac{2(s+1)}{3}}\left(\int_{-\infty}^{\infty} \frac{1}{1+\left|\tau-\left(\xi^{3}-\rho \xi\right)\right|+\xi^{2}} \hat{f}(\xi, \tau) d \xi\right)^{2} d \tau \\
& \leqslant C \int_{-\infty}^{\infty}(1+|\tau|)^{\frac{2(s+1)}{3}} G(\tau) \int_{-\infty}^{\infty} \frac{|\hat{f}(\xi, \tau)|^{2}\langle\xi\rangle^{2 s}}{\left(1+\left|\tau-\left(\xi^{3}-\rho \xi\right)\right|+\xi^{2}\right)^{2 b}} d \xi d \tau
\end{aligned}
$$

where

$$
\begin{aligned}
G(\tau) & :=\int_{-\infty}^{\infty} \frac{d \xi}{\langle\xi\rangle^{2 s}\left(1+\left|\tau-\left(\xi^{3}-\rho \xi\right)\right|+\xi^{2}\right)^{2(1-b)}} \\
& \leqslant C \int_{-\infty}^{\infty} \frac{d \eta}{|\eta|^{\frac{2}{3}}(1+|\eta|)^{\frac{2 s}{3}}(1+|\tau-\eta|)^{2(1-b)}}
\end{aligned}
$$

Therefore, it suffices to show that there exists a constant $C$ such that for any $\tau \in \Re$,

$$
G(\tau) \leqslant \frac{C}{(1+|\tau|)^{\frac{2(s+1)}{3}}} .
$$

To see this true, write $G(\tau)$ as

$$
G(\tau)=G_{1}(\tau)+G_{2}(\tau)+G_{3}(\tau)
$$

with

$$
\begin{aligned}
& G_{1}(\tau)=\int_{|\eta| \leqslant \frac{1}{2}|\tau|} \frac{d \eta}{|\eta|^{\frac{2}{3}}(1+|\eta|)^{\frac{2 s}{3}}(1+|\tau-\eta|)^{2(1-b)}}, \\
& G_{2}(\tau)=\int_{|\tau| / 2<|\eta|<2|\tau|} \frac{d \eta}{|\eta|^{\frac{2}{3}}(1+|\eta|)^{\frac{2 s}{3}}(1+|\tau-\eta|)^{2(1-b)}}
\end{aligned}
$$

and

$$
G_{3}(\tau)=\int_{2|\tau| \leqslant|\eta|} \frac{d \eta}{|\eta|^{\frac{2}{3}}(1+|\eta|)^{\frac{2 s}{3}}(1+|\tau-\eta|)^{2(1-b)}} .
$$

In the region $2|\eta| \leqslant|\tau|$, note that $(1+|\tau-\eta|) \geqslant \frac{1}{2}(1+|\tau|)$ and $|\tau-\eta| \geqslant \frac{1}{2}|\eta|$. Thus, for $s \leqslant 0$, 


$$
\begin{aligned}
G_{1}(\tau) & \leqslant C(1+|\tau|)^{-\frac{2(s+1)}{3}} \int_{-\infty}^{\infty} \frac{d \eta}{|\eta|^{\frac{2}{3}}(1+|\eta|)^{2(1-b)-\frac{2}{3}}} \\
& \leqslant \frac{C}{(1+|\tau|)^{\frac{2(s+1)}{3}}}
\end{aligned}
$$

as $b<\frac{1}{2}$. When $0 \leqslant s \leqslant 2-3 b$,

$$
\begin{aligned}
G_{1}(\tau) & \leqslant C(1+|\tau|)^{-\frac{2(s+1)}{3}} \int_{-\infty}^{\infty} \frac{d \eta}{|\eta|^{\frac{2}{3}}(1+|\eta|)^{\frac{2 s}{3}}(1+|\tau-\eta|)^{2(1-b)-\frac{2(s+1)}{3}}} \\
& \leqslant C(1+|\tau|)^{-\frac{2(s+1)}{3}} \int_{-\infty}^{\infty} \frac{d \eta}{|\eta|^{\frac{2}{3}}(1+|\eta|)^{2(1-b)-\frac{2}{3}}} \\
& \leqslant \frac{C}{(1+|\tau|)^{\frac{2(s+1)}{3}}} .
\end{aligned}
$$

In the region $|\tau| / 2<|\eta|<2|\tau|$,

$$
G_{2}(\tau) \leqslant C(1+|\tau|)^{-\frac{2(s+1)}{3}} \int_{|\tau| / 2}^{2|\tau|} \frac{d \eta}{(1+|\tau-\eta|)^{2(1-b)}} \leqslant \frac{C}{(1+|\tau|)^{2 \frac{s+1}{3}}},
$$

again because $b<1 / 2$. In the region $2|\tau| \leqslant|\eta|$, it is the case that $1+|\tau-\eta|=O(1+|\eta|)$. Thus, it transpires that

$$
G_{3}(\tau) \leqslant C \int_{|\eta| \geqslant 2|\tau|} \frac{d \eta}{|\eta|^{\frac{2(s+1)}{3}}(1+|\eta|)^{2(1-b)}} \leqslant C(1+|\tau|)^{-\frac{2(s+1)}{3}} .
$$

The inequality (2.20) has therefore been established for $-1 \leqslant s \leqslant 2-3 b$. This completes the proof.

Finally, attention is focused upon the boundary integral operators $\mathcal{B I}_{1}(t)$ and $\mathcal{B I}_{2}(t)$.

Proposition 2.8. Let $\psi \in C_{0}^{\infty}(\Re)$ be given and assume that $0 \leqslant b<\frac{1}{2}-\frac{s}{3}$ with $s \leqslant 0$. Then there exists a constant $C$ such that

$$
\left\|\psi \mathcal{B} \mathcal{I}_{1}(h)\right\|_{X_{s, b}} \leqslant C\|h\|_{H} \frac{3 b+s-1 / 2}{3}\left(\Re^{+}\right)
$$

for any $h \in H_{0}^{\frac{3 b+s-1 / 2}{3}}\left(\Re^{+}\right)$.

Proposition 2.9. Let $\psi \in C_{0}^{\infty}(\Re)$ be given and assume that $0 \leqslant b<\frac{5}{6}-\frac{s}{3}$ with $-\frac{1}{2}<s<1$. Then there exists a constant $C$ such that

$$
\left\|\psi \mathcal{B} \mathcal{I}_{2}(h)\right\|_{X_{s, b}} \leqslant C\|h\|_{H} \frac{3 b+s-1 / 2}{3}\left(\Re^{+}\right)
$$

for any $h \in H_{0}^{\frac{3 b+s-1 / 2}{3}}\left(\Re^{+}\right)$.

Proposition 2.10. Let $-\frac{3}{2}<\alpha<\frac{1}{2}$ and $-\frac{1}{2}<\beta<1$ be given. There exist constants $C_{\alpha}$ and $C_{\beta}$ such that

$$
\sup _{t \in R}\left\|\mathcal{B} \mathcal{I}_{1} h\right\|_{H^{\alpha}(\mathfrak{R})} \leqslant C_{\alpha}\|h\|_{H^{\frac{\alpha+1}{3}}\left(\mathfrak{R}^{+}\right)}
$$

and

$$
\sup _{t \in R}\left\|\mathcal{B I} \mathcal{I}_{2} h\right\|_{H^{\beta}(\Re)} \leqslant C_{\beta}\|h\|_{H^{\frac{\beta+1}{3}}\left(\Re^{+}\right)} .
$$


Observe that

$$
\|w\|_{L^{2}\left(0, T ; H^{s}(\Re)\right)} \leqslant C \Lambda_{s, b}(\psi w)
$$

for any $s \in \Re$ and $b \geqslant 0$, if $\psi \in C_{0}^{\infty}(\Re)$ and $\psi(t)=1$ when $t \in(0, T)$. The following result, which follows from Propositions 2.8 and 2.9, presents a boundary smoothing property of the linear KdV-Burgers equation, which is the same as that obtained for the linear KdV equation in [12].

Corollary 2.11. For any given $T>0$ and $s \geqslant-\frac{3}{2}$, there exists a constant $C$ such that

$$
\left\|\mathcal{W}_{b d r} h\right\|_{L^{2}\left(0, T ; H^{\left.s+\frac{3}{2}\left(\Re^{+}\right)\right)}\right.} \leqslant C\|h\|_{H^{\frac{1+s}{3}}\left(\Re^{+}\right)}
$$

for any $h \in H_{0}^{\frac{1+s}{3}}\left(\Re^{+}\right)$.

The boundary integral operator $\mathcal{W}_{b d r}$ also possesses the sharp Kato smoothing property, the final result of this section.

Proposition 2.12. For any given $T>0$ and $s \geqslant-\frac{3}{2}$, there exists a constant $C$ such that

$$
\sup _{x \in \Re^{+}}\left\|\partial_{x} \mathcal{W}_{b d r} h\right\|_{H_{t}^{\frac{s}{3}}\left(\Re^{+}\right)} \leqslant C\|h\|_{H^{\frac{s+1}{3}}\left(\Re^{+}\right)}
$$

for any $h \in H_{0}^{\frac{1+s}{3}}\left(\Re^{+}\right)$.

The proofs of Propositions 2.8-2.10 are similar to the results proved in Section 3 of [12]. A sketch of the proofs of Propositions 2.8 and 2.9 is provided in Appendix A for the reader's convenience. As for Proposition 2.12, its proof is the same as the analogous result in [12] and is therefore omitted.

\section{Well-posedness}

In this section, we study the well-posedness of the IBVP

$$
\left.\begin{array}{ll}
u_{t}+\rho u_{x}+\gamma u u_{x}+a_{1}(x) b_{1}(t) u u_{x}+a_{2}(x) b_{2}(t) u^{2}+u_{x x x}-u_{x x}=0, & x, t>0, \\
u(x, 0)=\phi(x), & x \geqslant 0, \\
u(0, t)=h(t), & t \geqslant 0,
\end{array}\right\}
$$

where $\rho>0$ and $\gamma \in R$ are constants, $a_{j} \in H^{\infty}\left(\Re^{+}\right)$and $b_{j} \in C^{\infty}\left(\Re^{+}\right)$for $j=1,2$.

As mentioned earlier, for given $s \in \Re$ and $0 \leqslant b \leqslant 1, X_{s, b}$ is the space of all distributions $w$ satisfying

$$
\|w\|_{X_{s, b}}:=\Lambda_{s, b}(w)<\infty
$$

(see (2.14)) and

$$
\mathcal{X}_{s, b} \equiv C\left(R ; H^{s}(R)\right) \cap X_{s, b} .
$$

In addition, for given $T>0$, let $\mathcal{D}_{s, T}$ be the space of all pairs $(\phi, h) \in H^{s}\left(\Re^{+}\right) \times H^{\frac{s+1}{3}}(0, T)$ which are $s$-compatible with respect to the $\operatorname{IBVP}(3.1)$, with norm inherited from the space $H^{s}\left(\Re^{+}\right) \times H^{\frac{s+1}{3}}(0, T)$, i.e.,

$$
\|(\phi, h)\|_{\mathcal{D}_{s, T}}:=\|(\phi, h)\|_{H^{s}\left(\Re^{+}\right) \times H^{\frac{s+1}{3}}(0, T)}
$$

for any $(\phi, h) \in \mathcal{D}_{s, T}$. Let $\mathcal{Y}_{s, b}$ be the space of all functions $w$ in $\mathcal{X}_{s, b}$ satisfying

$$
\sup _{x \in \mathfrak{R}}\left\|w_{x}(x, \cdot)\right\|_{H_{t}^{\frac{s}{3}}(\mathfrak{R})}<+\infty .
$$

For any $w \in \mathcal{Y}_{s, b}$, define

$$
\|w\| \mathcal{Y}_{s, b}=\left(\|w\|_{\mathcal{X}_{s, b}}^{2}+\sup _{x \in \mathfrak{R}}\left\|w_{x}(x, \cdot)\right\|_{H_{t}^{\frac{s}{3}}(\Re)}^{2}\right)^{\frac{1}{2}} .
$$


These Bourgain-type spaces are defined for distributions whose domain is the whole plane $\Re \times \Re$. However, the IBVP (3.1) is posed on the quarter plane $\mathfrak{R}^{+} \times \Re^{+}$and we are seeking its solution in the space $C\left(\Re^{+}: H^{s}\left(\Re^{+}\right)\right)$ corresponding to a given initial value in the space $H^{s}\left(\Re^{+}\right)$and boundary data in the space $H_{\text {loc }}^{\frac{s+1}{3}}\left(\Re^{+}\right)$. It is thus natural to consider versions of the these Bourgain-type spaces restricted to the quarter plane $\mathfrak{R}^{+} \times \mathfrak{R}^{+}$. Let $\Omega$ denote a subinterval of $\Re$; define a Bourgain space $X_{s, b}$ restricted to the domain $\mathfrak{R}^{+} \times \Omega$ as follows:

$$
X_{s, b}\left(\Re^{+} \times \Omega\right)=\left.X_{s, b}\right|_{\Re^{+} \times \Omega}
$$

with the quotient norm

$$
\|u\|_{X_{s, b}\left(\Re^{+} \times \Omega\right)} \equiv \inf _{w \in X_{s, b}}\left\{\|w\|_{X_{s, b}}: w(x, t)=u(x, t) \text { on } \Re^{+} \times \Omega\right\} .
$$

The spaces $\mathcal{X}_{s, b}\left(\Re^{+} \times \Omega\right)$ and $\mathcal{Y}_{s, b}\left(\Re^{+} \times \Omega\right)$ are defined similarly.

For the IBVP (3.1), the following well-posedness result obtains.

Theorem 3.1. Let $s \in\left(-1, \frac{1}{2}\right)$ and $r>0$ be given. There exists $T$ with $T>0$ such that for a given pair $(\phi, h) \in \mathcal{D}_{s, T}$ satisfying

$$
\|(\phi, h)\|_{\mathcal{D}_{s, T}} \leqslant r
$$

the IBVP (3.1) admits a unique solution $u \in \mathcal{Y}_{s, \frac{1}{2}}\left(\Re^{+} \times(0, T)\right)$. Moreover, the solution $u$ depends Lipschitz continuously on $(\phi, h)$ in the corresponding spaces.

The proof of Theorem 3.1 is based on the results in Section 2 and the following lemmas.

Consider the non-homogeneous linear problem

$$
\left.\begin{array}{l}
u_{t}+\rho u_{x}-u_{x x}+u_{x x x}=0, \quad \text { for } x, t \geqslant 0, \\
u(x, 0)=0, \quad u(0, t)=h(t) .
\end{array}\right\}
$$

Recall its solution may be written in the form

$$
u(x, t)=\left[\mathcal{W}_{b d r}(t) h\right](x)
$$

for $x, t \geqslant 0$, as expounded in Section 2.

Lemma 3.2. For a given pair $(b, s)$ satisfying

$$
\begin{cases}0 \leqslant b<\frac{1}{2}-\frac{s}{3} & \text { if } s \leqslant 0 \\ 0 \leqslant b<\frac{5}{6}-\frac{s}{3} & \text { if }-\frac{1}{2}<s<1 \\ b=\frac{1}{2} & \text { if } 1 \leqslant s \leqslant 3\end{cases}
$$

there exists a constant $C$ such that for any $T>0$ and any $h \in H_{0}^{\frac{s+1}{3}}(0, T)$, the corresponding solution $u$ of (3.2) belongs to the restricted Bourgain space $\mathcal{Y}_{s, b}\left(\Re^{+} \times(0, T)\right)$ and satisfies the bounds

$$
\begin{aligned}
& \|u\|_{X_{s, b}\left(\Re^{+} \times(0, T)\right)} \leqslant C\|h\|_{H}{ }_{H}^{\frac{3 b+s-1 / 2}{3}}(0, T) \\
& \|u\|_{\mathcal{X}_{s, \frac{1}{2}}\left(\Re^{+} \times(0, T)\right)} \leqslant C\|h\|_{H^{\frac{s+1}{3}}(0, T)}
\end{aligned}
$$

and

$$
\|u\|_{\mathcal{Y}_{s, \frac{1}{2}}\left(\Re^{+} \times(0, T)\right)} \leqslant C\|h\|_{H^{\frac{s+1}{3}}(0, T)} .
$$

Proof. For $T>0$, let $h_{1} \in H^{\frac{s+1}{3}}\left(\Re^{+}\right)$such that $h_{1} \equiv h$ in the space $H^{\frac{s+1}{3}}(0, T)$ and let $\psi_{1} \in C_{0}^{\infty}(R)$ be such that $\psi_{1}(t)=1$ for all $t \in[0, T]$. Define

$$
u_{1}(x, t)= \begin{cases}{\left[\mathcal{B I}_{1}(t) h_{1}\right](x)} & \text { if } 0 \leqslant b<\frac{1}{2}-\frac{s}{3} \text { and } s<0, \\ {\left[\mathcal{B I}_{2}(t) h_{1}\right](x)} & \text { if } 0 \leqslant b<\frac{5}{6}-\frac{s}{3} \text { and } 0 \leqslant s<1 .\end{cases}
$$


Observing that

$$
u(x, t)=u_{1}(x, t) \quad \text { for }(x, t) \in \mathfrak{R}^{+} \times[0, T],
$$

and using Propositions 2.8-2.10, one arrives at the inequalities

$$
\|u\|_{\mathcal{X}_{s, \frac{1}{2}}\left(\Re^{+} \times(0, T)\right)} \leqslant\left\|\psi_{1} u_{1}\right\|_{\mathcal{X}_{s, \frac{1}{2}}} \leqslant C\left\|h_{1}\right\|_{H^{\frac{s+1}{3}}\left(\Re^{+}\right)}
$$

from which (3.5) with $-1<s<1$ follows. In particular,

$$
\|u\|_{\mathcal{X}_{0, \frac{1}{2}}\left(\Re^{+} \times(0, T)\right)} \leqslant C\|h\|_{H^{\frac{1}{3}}\left(\Re^{+}\right)} .
$$

When $1 \leqslant s \leqslant 3$, let $v=u_{t}$. Then $v$ solves

$$
\left.\begin{array}{l}
v_{t}+\rho v_{x}-v_{x x}+v_{x x x}=0, \quad \text { for } x, t \geqslant 0, \\
v(x, 0)=0, \quad v(0, t)=h^{\prime}(t) .
\end{array}\right\}
$$

Thus

$$
\|v\|_{\mathcal{X}_{0, \frac{1}{2}}\left(\Re^{+} \times(0, T)\right)} \leqslant C\left\|h^{\prime}\right\|_{H^{\frac{1}{3}\left(\Re^{+}\right)}} \leqslant c\|h\|_{H^{\frac{4}{3}}\left(\Re^{+}\right)} .
$$

Since $v=u_{t}=-\rho u_{x}+u_{x x}-u_{x x x}$, it follows that

$$
\|u\|_{\mathcal{X}_{3, \frac{1}{2}}\left(\Re^{+} \times(0, T)\right)} \leqslant C\|h\|_{H^{\frac{4}{3}}\left(\Re^{+}\right)} .
$$

The inequality (3.5) with $1 \leqslant s \leqslant 3$ follows by interpolation of the estimates (3.7) and (3.8). The proof is complete.

Consider the same linear equation posed with zero boundary conditions, but non-trivial initial data, viz.

$$
\left.\begin{array}{l}
u_{t}+\rho u_{x}-u_{x x}+u_{x x x}=0, \quad \text { for } x, t \geqslant 0, \\
u(x, 0)=\phi(x), \quad u(0, t)=0 .
\end{array}\right\}
$$

As mentioned earlier, its solution can be written as

$$
u(x, t)=\left[\mathcal{W}_{c}(t) \phi\right](x)
$$

for $x, t \geqslant 0$.

Lemma 3.3. For a given $s \in\left(-1, \frac{1}{2}\right)$, there exists a constant $C$ such that for any $T>0$ and any $\phi \in H_{0}^{s}\left(\Re^{+}\right)$, the corresponding solution $u$ of (3.9) belongs to the restricted Bourgain space $\mathcal{Y}_{s, \frac{1}{2}}\left(\Re^{+} \times(0, T)\right)$ and satisfies the inequality

$$
\|u\|_{\mathcal{Y}_{s, \frac{1}{2}}\left(\Re^{+} \times(0, T)\right)} \leqslant C\|\phi\|_{H^{s}\left(\Re^{+}\right)} .
$$

Proof. According to Proposition 2.2, one may write $W_{c}(t) \phi$ as

$$
\mathcal{W}_{c}(t) \phi=W_{\Re}(t) \phi^{*}-\mathcal{W}_{b d r}(t) g
$$

for any $x, t>0$, where $g$ is the trace of $W_{\Re}(t) \phi^{*}$ at $x=0, \phi^{*} \in H^{s}(\Re)$ and $\phi^{*}$ equals $\phi$ when restricted on $\Re^{+}$. The estimate (3.10) follows from Propositions 2.4, 2.5 and Lemma 3.2.

We turn now to consideration of the forced linear problem

$$
\left.\begin{array}{l}
u_{t}+\rho u_{x}-u_{x x}+u_{x x x}=f, \quad \text { for } x, t \geqslant 0, \\
u(x, 0)=0, \quad u(0, t)=0 .
\end{array}\right\}
$$

Its solution can be written in the form

$$
u(\cdot, t)=\int_{0}^{t} \mathcal{W}_{c}(t-\tau) f(\cdot, \tau) d \tau .
$$


Lemma 3.4. Assume that $-1<s \leqslant 3-2 b$ and $0<b<\frac{1}{2}$. There exists a constant $C$ such that for any $T>0$ and any $f \in X_{s,-b}\left(\Re^{+} \times(0, T)\right)$, the corresponding solution $u$ of $(3.11)$ belongs to the space $\mathcal{X}_{s, 1 / 2}\left(\Re^{+} \times(0, T)\right)$ and satisfies the estimate

$$
\|u\|_{\mathcal{Y}_{s, \frac{1}{2}}\left(\Re^{+} \times(0, T)\right)} \leqslant C\|f\|_{X_{s,-b}(\Re+\times(0, T))} .
$$

Proof. By Proposition 2.3,

$$
u(\cdot, t)=\int_{0}^{t} W_{\Re}(t-\tau) f(\cdot, \tau) d \tau-\mathcal{W}_{b d r}(t) v
$$

for any $x, t>0$ where $v \equiv v(t)$ is the trace of $\int_{0}^{t} W_{\mathfrak{R}}(t-\tau) f(\cdot, \tau) d \tau$ at $x=0$. The estimate (3.12) then follows from Propositions 2.4, 2.7 and Lemma 3.2.

The next lemma presents a version of so-called bilinear estimates in the restricted Bourgain space $X_{s, b}\left(\Re^{+} \times\right.$ $(0, T))$ which follows directly from Lemma 3.1 in [47].

Lemma 3.5. Given $s>-1$ and $T>0$, there exist positive constants $C, \mu, \delta$ such that

$$
\left\|\partial_{x}(u v)\right\|_{X_{s,-1 / 2+\delta}\left(\Re^{+} \times(0, T)\right)} \leqslant C T^{\mu}\|u\|_{X_{s, 1 / 2}\left(\Re^{+} \times(0, T)\right)}\|v\|_{X_{s, 1 / 2}\left(\Re^{+} \times(0, T)\right)}
$$

for any $u, v \in X_{s, 1 / 2}\left(\Re^{+} \times(0, T)\right)$.

We are now prepared to present a proof of Theorem 3.1.

Proof of Theorem 3.1. By applying Lemmas 3.2-3.5, Theorem 3.1 can be established by the standard contraction mapping principle.

Let $\phi \in H^{s}\left(\Re^{+}\right)$and $h \in H_{\text {loc }}^{\frac{s+1}{3}}\left(\Re^{+}\right)$be given with $s \in\left(-1, \frac{1}{2}\right)$. For given $\theta$ with $0<\theta \leqslant 1$ (to be chosen precisely momentarily) and $v, w \in \mathcal{Y}_{s, \frac{1}{2}}\left(\Re^{+} \times(0, \theta)\right)$, define

$$
\mathbf{F}(w)=W_{c}(t) \phi+\mathcal{W}_{b d r}(t) h-\int_{0}^{t} \mathcal{W}_{c}(t-\tau)\left(a_{1} b_{1} w_{x} w+a_{2} b_{2} w^{2}\right)(\tau) d \tau .
$$

Using Lemmas 3.2-3.5, it is seen that

$$
\|\mathbf{F}(w)\|_{\mathcal{Y}_{s, \frac{1}{2}}\left(\Re^{+} \times(0, \theta)\right)} \leqslant C_{1}\left(\|\phi\|_{H^{s}\left(\Re^{+}\right)}+\|h\|_{H^{\frac{s+1}{3}}(0, T)}\right)+C_{2} \theta^{\mu}\|w\|_{\mathcal{Y}_{s, \frac{1}{2}}\left(\Re^{+} \times(0, \theta)\right)}
$$

and

$$
\|\mathbf{F}(v)-\mathbf{F}(w)\|_{\mathcal{Y}_{s, \frac{1}{2}}\left(\Re^{+} \times \theta\right)} \leqslant C_{2} \theta^{\mu}\|v-w\|_{\mathcal{Y}_{s, \frac{1}{2}}\left(\Re^{+} \times(0, \theta)\right)}\|v+w\|_{\mathcal{Y}_{s, \frac{1}{2}}\left(\Re^{+} \times(0, \theta)\right)}
$$

where the constants $C_{1}$ and $C_{2}$ are independent of $\theta, v$ and $w$. Let $B_{r}$ be the ball centered at the origin in the space $\mathcal{Y}_{s, \frac{1}{2}}\left(\Re^{+} \times(0, \theta)\right)$ with radius $r$ where

$$
r=2 C_{1}\left(\|\phi\|_{H^{s}\left(\Re^{+}\right)}+\|h\|_{H^{\frac{s+1}{3}}(0, \theta)}\right),
$$

and choose $\theta=T$ small enough that

$$
2 C_{2} T^{\mu} r \equiv \beta<1 .
$$

In this case, it follows readily that $\mathbf{F}$ maps $B_{r}$ into itself and that for $w, v \in B_{r}$,

$$
\|\mathbf{F}(w)-\mathbf{F}(v)\|_{\mathcal{Y}_{s, \frac{1}{2}}\left(\Re^{+} \times(0, T)\right)} \leqslant \beta\|w-v\|_{\mathcal{Y}_{s, \frac{1}{2}}\left(\Re^{+} \times(0, T)\right)}
$$


Thus, the mapping $\mathbf{F}$ is a contraction mapping of the ball $B_{r}$. The fixed point $u$ in $B_{r}$ of this map $\mathbf{F}$ is the advertised solution.

The well-posedness result presented in Theorem 3.1 is conditional since uniqueness is established in the space $\mathcal{Y}_{s, \frac{1}{2}}\left(\Re^{+} \times(0, T)\right)$ instead of in the space $C\left([0, T] ; H^{s}\left(\Re^{+}\right)\right)$. However, following the procedure developed in [11], one can show that in fact, uniqueness holds in the space $C\left([0, T] ; H^{s}\left(\Re^{+}\right)\right)$.

Proposition 3.6. Let $s \in\left(-1, \frac{1}{2}\right)$ and $r>0$ be given. There exists a $T>0$ depending only on $s$ and $r$ such that for a given $(\phi, h) \in \mathcal{D}_{s, T}$ satisfying

$$
\|\phi\|_{\mathcal{D}_{s, T}} \leqslant r
$$

the IBVP (3.1) admits a unique solution $u \in C\left([0, T] ; H^{s}\left(\Re^{+}\right)\right)$. Moreover, the solution $u$ depends Lipschitz continuously in the space $C\left([0, T] ; H^{s}\left(\Re^{+}\right)\right)$on $(\phi, h)$ in the space $\mathcal{D}_{s, T}$.

This well-posedness result can be extended to be valid for any $s \geqslant \frac{1}{2}$.

Theorem 3.7. Let $s>-1$ and $r>0$ be given with $s \neq 3 m+\frac{1}{2}, m=0,1,2, \ldots$ There exists a $T>0$ depending only on $s$ and $r$ such that for a given $(\phi, h) \in \mathcal{D}_{s, T}$ satisfying

$$
\|\phi\|_{\mathcal{D}_{s, T}} \leqslant r
$$

the IBVP (3.1) admits a unique solution $u \in C\left([0, T] ; H^{s}\left(\Re^{+}\right)\right)$. Moreover, the solution u depends Lipschitz continuously on $(\phi, h)$ in their respective spaces.

Proof. The proof is provided only for the case wherein $0 \leqslant s \leqslant 3$. The proof of the remaining case is similar and so is omitted.

First note that according to Theorem 3.1, for a given $r_{0}>0$, there exists a $T_{0}>0$ depending only on $r_{0}$ such that if

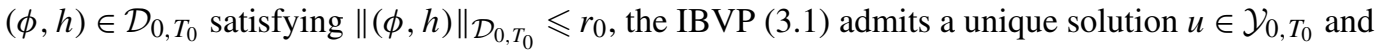

$$
\|u\|_{\mathcal{Y}_{0, T_{0}}} \leqslant C_{r_{0}}\|(\phi, h)\|_{\mathcal{D}_{0, T_{0}}}
$$

where $C_{r_{0}}$ is a constant which depends only on $r_{0}$. (This dependence can be taken to be continuous if we like.) Moreover, for any $\left(\phi_{j}, h_{j}\right) \in \mathcal{D}_{0, T_{0}}$ with $\left\|\left(\phi_{j}, h_{j}\right)\right\|_{\mathcal{D}_{0, T_{0}}} \leqslant r_{0}$ for $j=1,2$, the corresponding solutions $u_{j}, j=1,2$, to the IBVP (3.1) satisfy

$$
\left\|u_{1}-u_{2}\right\| \mathcal{Y}_{0, T_{0}} \leqslant C_{r_{0}}\left\|\left(\phi_{1}, h_{1}\right)-\left(\phi_{2}, h_{2}\right)\right\|_{\mathcal{D}_{0, T_{0}}} .
$$

Next suppose given $(\phi, h) \in \mathcal{D}_{3, T_{0}}$ satisfying

$$
\|(\phi, h)\|_{\mathcal{D}_{0, T_{0}}} \leqslant r_{0} \text {. }
$$

Applying Theorem 3.1 gives a solution $u \in \mathcal{Y}_{0, T_{0}}$ satisfying the estimate (3.14). We claim this solution $u$, in fact, belongs to the space $C\left(\left[0, T_{0}\right] ; H^{3}\left(\Re^{+}\right)\right)$and

$$
\|u\|_{C\left(\left[0, T_{0}\right] ; H^{3}\left(\Re^{+}\right)\right)} \leqslant C_{r_{0}}\|(\phi, h)\|_{\mathcal{D}_{3, T_{0}}} .
$$

To see this, let $v=u_{t}$. Then $v$ satisfies

$$
\left.\begin{array}{l}
v_{t}+\rho v_{x}+\left(\gamma+a_{1} b_{1}\right)(u v)_{x}+2 a_{2} b_{2} u v-v_{x x}+v_{x x x}=f, \quad x, t \in \Re^{+}, \\
v(x, 0)=\phi_{1}(x), \quad v(0, t)=h_{1}(t) .
\end{array}\right\}
$$

Here, $f=-a_{1}(x) b_{1}^{\prime}(t) u u_{x}-a_{2}(x) b_{2}^{\prime}(t) u^{2}, h_{1}(t)=h^{\prime}(t)$ and $\phi_{1}$ is defined as in (1.2). This is an IBVP for a linearized $\mathrm{KdV}$-Burgers equation with variable coefficients. The same argument as presented in support of Theorem 3.1 yields that there exists a $T^{\prime} \in\left(0, T_{0}\right]$ such that the IBVP (3.15) has a unique solution $v \in \mathcal{Y}_{0, T^{\prime}}$ since $\phi_{1} \in L^{2}\left(\Re^{+}\right)$and $h_{1} \in H^{\frac{1}{3}}\left(0, T_{0}\right)$. This, in turn, implies that $u \in C\left(\left[0, T^{\prime}\right] ; H^{3}\left(\Re^{+}\right)\right)$since

$$
v=u_{t}=-u_{x x x}+u_{x x}-\gamma u u_{x}-\rho u_{x}-a_{1}(x) b_{1}(t) u u_{x}-a_{2}(x) b_{2}(t) u^{2} .
$$


Note that $T^{\prime}$ only depends on $\|u\|_{\mathcal{Y}_{0, T_{0}}}$ and therefore only on $r_{0}$ according to (3.14). By a standard argument, one may extend $T^{\prime}$ so that $T^{\prime}=T_{0}$.

Thus, Theorem 3.7 holds for $s=0$ and $s=3$. To see it is also true for $0<s<3$, for any $(\phi, h) \in \mathcal{D}_{s, T_{0}}$, define

$$
I_{r_{0}}(\phi, h)= \begin{cases}(\phi, h) & \text { if }\|(\phi, h)\|_{\mathcal{D}_{0, T_{0}} \leqslant r_{0},} \\ \frac{r}{\|(\phi, h)\|_{\mathcal{D}_{0, T_{0}}}}(\phi, h) & \text { if }\|(\phi, h)\|_{\mathcal{D}_{0, T_{0}} \geqslant r_{0} .}\end{cases}
$$

Then, the IBVP (3.1) defines a non-linear map $K$ from $\mathcal{D}_{j, T_{0}}$ to the space $C\left([0, T] ; H^{j}\left(\Re^{+}\right)\right)$for $j=0,3$ by

$$
K(\phi, h)=u
$$

where $u$ is the solution of the IBVP (3.1) with $(\phi, h)$ replaced by $I_{r}(\phi, h)$. Moreover, $K$ is Lipschitz continuous from the space $\mathcal{D}_{0, T_{0}}$ to the space $C\left(\left[0, T_{0}\right] ; L^{2}\left(\Re^{+}\right)\right)$and there exists a constant $C^{*}$ depending only on $\|(\phi, h)\|_{\mathcal{D}_{0, T_{0}}}$ such that

$$
\|K(\phi, h)\|_{C\left(\left[0, T_{0}\right] ; H^{3}\left(\mathfrak{R}^{+}\right)\right)} \leqslant C^{*}\|(\phi, h)\|_{\mathcal{D}_{0, T_{0}}}
$$

for any $(\phi, h) \in \mathcal{D}_{3, T_{0}}$. Invoking non-linear interpolation theory as in [52], it is deduced that the operator $K$ is also well defined from the space $\mathcal{D}_{s, T_{0}}$ to the space $C\left(\left[0, T_{0}\right] ; H^{s}\left(\Re^{+}\right)\right)$for any $s \in(0,3)$. Thus, for any $(\phi, h) \in \mathcal{D}_{\int, \mathcal{T}_{1}}$

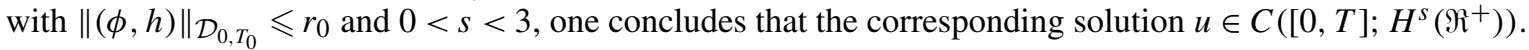

Next, attention is given to the issue of global well-posedness of the IBVP (3.1). The arguments used in [12] can be applied here to give the following global well-posedness result.

Theorem 3.8. Let $s \geqslant 0$ and $T>0$ be given with $s \neq 3 m+\frac{1}{2}, m=0,1,2, \ldots$ Assume that the coefficients $a_{1}(x) b_{1}(t)$ and $a_{2}(x) b_{2}(t)$ in the system (3.1) satisfy

$$
a_{2}(x) b_{2}(t) \equiv \frac{1}{3} a_{1}^{\prime}(x) b_{1}(t) \quad \text { for any } t, x>0 .
$$

Then, for any s-compatible $(\phi, h) \in H^{s}\left(\Re^{+}\right) \times H_{\mathrm{loc}}^{\frac{s+1}{3}+\eta(s)}\left(\Re^{+}\right)$, the IBVP (3.1) admits a unique solution $u \in$ $C\left([0, T] ; H^{s}\left(\Re^{+}\right)\right)$, where $\eta(s)=0$ when $s \geqslant 3$ and $\eta(s)=\epsilon$ when $0 \leqslant s<3$ for an arbitrarily small $\epsilon>0$. Moreover, the solution $u$ depends analytically on $\phi$ and $h$ in their respective function classes.

Note that as a consequence of the assumption (3.16), the system (3.1) has the following global a priori estimate: any smooth solution $u$ of the IBVP (3.1) with boundary data $h \equiv 0$ satisfies

$$
\sup _{0 \leqslant t \leqslant T}\|u(\cdot, t)\|_{L^{2}\left(\Re^{+}\right)} \equiv\|\phi\|_{L^{2}\left(\Re^{+}\right)} .
$$

When $-1<s<0$, Molinet and Ribaud [47] showed that the IVP for the KdV-Burgers equation is also globally well-posed in the space $H^{s}(\Re)$ by taking the advantage of the dissipative term $-u_{x x}$. We have a similar global wellposedness result for the IBVP (3.1).

Theorem 3.9. Assume that the coefficients $a_{1}(x) b_{1}(t)$ and $a_{2}(x) b_{2}(t)$ in the system (3.1) satisfy condition (3.16). Let $-1<s<0$ and $T>0$ be given. For any given $(\phi, h) \in H^{s}\left(\Re^{+}\right) \times H_{\mathrm{loc}}^{\frac{1^{+}}{3}}\left(\Re^{+}\right)$, the IBVP (3.1) admits a unique solution $u \in C\left([0, T] ; H^{s}\left(\Re^{+}\right)\right)$. Moreover, for any $\epsilon>0$, this solution also lies in the class $L^{2}\left([\epsilon, T] ; H^{\frac{3}{2}}\left(\Re^{+}\right)\right)$.

The following lemma is needed in the proof.

Lemma 3.10. Suppose to be given $s>-1$ and $T>0$. There exists a $\delta>0$ such that for all $u \in \mathcal{X}_{s, \frac{1}{2}}\left(\Re^{+} \times(0, T)\right)$,

$$
w=\int_{0}^{t} \mathcal{W}_{c}(t-\tau)\left(u u_{x}\right)(\tau) d \tau \in L^{2}\left(0, T ; H^{s+\delta}\left(\Re^{+}\right)\right) .
$$


Proof. By Proposition 2.3, we may rewrite $w$ as $w=w_{1}+w_{2}$ with

$$
w_{1}=\int_{0}^{t} \mathcal{W}_{\Re}(t-\tau)\left(u u_{x}\right)(\tau) d \tau \quad \text { and } \quad w_{2}=\mathcal{W}_{b d r}(t) g
$$

where $g$ is the trace of $w_{1}$ at $x=0$. According to Lemma 3.5, there exists a $\delta>0$ such that $u u_{x} \in \mathcal{X}_{s,-\frac{1}{2}+\delta}\left(\Re^{+} \times\right.$ $(0, T))$. Thus, $w_{1} \in L^{2}\left(0, T ; H^{s+\delta}\left(\Re^{+}\right)\right)$by Proposition 2.4. In addition, it follows from Proposition 2.5 that $g \in$ $H^{\frac{s+1}{3}}(\Re)$ which yields that $w_{2} \in L^{2}\left(0, T ; H^{s+\delta}\left(\Re^{+}\right)\right)$by Corollary 2.11. The proof is complete.

Proof of Theorem 3.9. By Theorem 3.1, the IBVP (3.1) admits a unique solution

$$
u \in C\left(\left[0, T^{*}\right] ; H^{s}\left(\Re^{+}\right)\right)
$$

for some $T^{*} \leqslant T$. Moreover, $u$ can be decomposed in the form

$$
u(x, t)=u_{1}(x, t)+u_{2}(x, t)+u_{3}(x, t)
$$

with

$$
u_{1}(x, t)=\mathcal{W}_{c}(t) \phi, \quad u_{2}(x, t)=\mathcal{W}_{b r d}(t) h, \quad u_{3}(x, t)=-\int_{0}^{t} \mathcal{W}_{c}(t-\tau)\left(a_{1} b_{1} u u_{x}+a_{2} b_{2} u^{2}\right)(\tau) d \tau .
$$

According to Corollary 2.11, $u_{2} \in L^{2}\left(0, T ; H^{\frac{3}{2}}\left(\Re^{+}\right)\right)$. By Proposition 2.2,

$$
u_{1}=W_{\Re}(t) \phi^{*}-\mathcal{W}_{b d r}(t) h_{1}
$$

with $h_{1}$ being the trace of $W_{R}(t) \phi^{*}$ at $x=0$. As remarked before, for any $\epsilon>0, u_{1} \in C\left([\epsilon, T] ; H^{\infty}\left(\Re^{+}\right)\right)$. As for $u_{3}$, it follows from Lemma 3.10 that $u_{3} \in L^{2}\left(0, T^{*} ; H^{s+\delta}\left(\Re^{+}\right)\right)$for some $\delta>0$. Consequently, for any $\epsilon_{1}$ with $0<\epsilon_{1} \leqslant T^{*}$, one can find a $t_{1} \in\left(0, \epsilon_{1}\right)$ such that $u\left(\cdot, t_{1}\right) \in H^{s+\delta}\left(\Re^{+}\right)$. Taking $\psi(x)=u\left(x, t_{1}\right)$ as a new initial value for the IBVP (3.1), it follows from the same argument that $u\left(\cdot, t_{2}\right) \in H^{s+2 \delta}\left(\Re^{+}\right)$for some $t_{1}<t_{2}<\epsilon_{1}$. Repeating this procedure, one eventually arrives at the conclusion that $u\left(\cdot, t^{\prime}\right) \in H^{\frac{3}{2}}\left(\Re^{+}\right)$) for some $0<t^{\prime}<\epsilon_{1}$. The proof is completed by invoking Theorem 3.8.

For the pure initial value problem (1.6), if $\psi \in H^{s}(\Re)$ for some $s>-1$, then the corresponding solution $u$ lies in $C\left([\epsilon, T] ; H^{\infty}(\Re)\right)$. There is a similar result for (3.1), which follows directly from Theorem 3.9.

Corollary 3.11. Let $s>-1$ and $T>0$ be given. Assume that $(\phi, h) \in H^{s}\left(\Re^{+}\right) \times H^{\infty}(0, T)$. Then, the corresponding solution of the IBVP (3.1) belongs to the space $C\left([\epsilon, T] ; H^{\infty}\left(\Re^{+}\right)\right)$for any $\epsilon$ with $0<\epsilon<T$.

\section{Acknowledgements}

JLB and SMS were partially supported by the National Science Foundation. BYZ was partially supported by the Charles Phelps Taft Memorial Fund at the University of Cincinnati.

\section{Appendix A}

In this appendix, the proofs of Propositions 2.8 and 2.9 are presented. Since the development is very similar to that appearing in [12], we content ourselves with sketches. The reader is referred to [12] for details.

Proof of Proposition 2.8. Recall that

$$
\left[\mathcal{B I}_{1}(t) h\right](x)=I_{1}(x, t)+I_{2}(x, t),
$$

where $I_{1}(x, t)$ is a function defined on the whole plane $\Re \times \Re$ and is, in fact, a $C^{\infty}$-smooth function of $x$ and $t$. For any $t \in \Re$, 


$$
\begin{aligned}
\left\|I_{1}(x, t)\right\|_{L_{x}^{2}(\Re)} & \leqslant C\left\|\left(3 \mu^{2}-\rho\right) \phi_{1}(\mu) \int_{0}^{\infty} e^{-i\left(\mu^{3}-\rho \mu\right) \xi} h(\xi) d \xi\right\|_{L_{\mu}^{2}(\Re)} \\
& \leqslant C\|h\|_{L^{2}(\Re+)}
\end{aligned}
$$

This type of inequality is also valid for $\partial_{x}^{j} \partial_{t}^{l} I_{1}$ for any $j, l \geqslant 0$. Thus, it is straightforward to see that if $h \in L^{2}\left(\Re^{+}\right)$, then

$$
\Lambda_{s, b}\left(\psi I_{1}\right) \leqslant C\|h\|_{L^{2}\left(\Re^{+}\right)}
$$

for any given $b \geqslant 0$ and $s \in \Re$ where the constant $C$ depends only on $\psi, b$ and $s$.

To analyze $I_{2}(x, t)$, remember that

$$
\mathcal{F}_{x, t}\left[I_{2}\right](\xi, \tau)=\hat{I}_{21}(\xi, \tau)+\hat{I}_{22}(\xi, \tau)
$$

where, for $|\xi|>1$,

$$
\begin{aligned}
& \hat{I}_{21}(\xi, \tau)=\mathcal{F}_{t}\left[\int_{0}^{\infty} E(x, t) \cos (x \xi) d x\right](1-\Theta(\xi, \tau))(1+\omega(\tau)), \\
& \hat{I}_{22}(\xi, \tau)=i C_{2} \int_{0}^{\infty}\left(\frac{1}{\xi-\eta}+\frac{1}{\xi+\eta}\right) \mathcal{F}_{t}\left[\int_{0}^{\infty} E(x, t) \cos (x \eta) d x\right](2 \Theta(\eta, \tau)+(1-\Theta(\eta, \tau))(1+\omega(\tau))) d \eta .
\end{aligned}
$$

Since the relevant estimates in the region $|\xi|<1$ are straightforward, in what follows it is always assumed that $|\xi| \geqslant 1$. First, consider the term

$$
\int_{-\infty}^{\infty} \int_{-\infty}^{\infty}\left\langle i\left(\tau-\left(\xi^{3}-\rho \xi\right)\right)+\xi^{2}\right\rangle^{2 b}\langle\xi\rangle^{-2 s}\left|\hat{I}_{21}(\xi, \tau)\right|^{2} d \xi d \tau
$$

Proposition A.1. Let $s \leqslant 0$ and $0<b<\frac{1}{2}-\frac{s}{3}$ be given. There exists a constant $C$ such that

$$
\left.\int_{-\infty}^{\infty} \int_{-\infty}^{\infty}\left\langle i\left(\tau-\left(\xi^{3}-\rho \xi\right)\right)+\xi^{2}\right\rangle^{2 b}\langle\xi\rangle^{2 s}\left|\hat{I}_{21}(\xi, \tau)\right|^{2} d \xi d \tau<C\|h\|_{H^{2}}^{2}{ }^{\frac{3 b+s-1 / 2}{3}}{ }_{\left(\Re^{+}\right.}\right)
$$

for any $h \in H^{\frac{3 b+s-1 / 2}{3}}\left(\Re^{+}\right)$.

Proof. According to (3.11),

$$
\mathcal{F}_{t}\left[\int_{0}^{\infty} E(x, t) \cos (x, \xi) d x\right]=\sum_{m=1}^{4} K_{1}(\xi, \lambda) \phi_{2}(\mu(\lambda)) \hat{h}(\lambda)+\sum_{m=1}^{4} K_{m 2}(\xi,-\lambda) \phi_{2}(\mu(-\lambda)) \hat{h}(-\lambda) .
$$

In the following, detailed analysis is given for terms containing $K_{21}$; the estimates for the other terms follow similar lines. Suppose $\xi \geqslant 0$ in what follows. The case $\xi<0$ is entirely analogous. Write

$$
A_{m 1}(\xi, \tau)=K_{m 1}(\xi, \tau) \phi_{2}(\mu(\tau)) \hat{h}(\tau), \quad m=1,2,3 .
$$

For fixed $s \leqslant 0$ and $b>0$, we have

$$
\begin{aligned}
& \int_{-\infty}^{\infty} \int_{0}^{\infty}\left\langle i\left(\tau-\left(\xi^{3}-\rho \xi\right)\right)+\xi^{2}\right\rangle^{2 b}\langle\xi\rangle^{2 s}\left|A_{21}(\xi, \tau)(1+\omega(\tau))(1-\Theta(\xi, \tau))\right|^{2} d \xi d \tau \\
& \quad \leqslant C \int_{-\infty}^{\infty} \phi_{2}(\mu(\tau))\left|\int_{0}^{\infty} h(s) e^{-i s \tau} d s\right|^{2} B_{21}(\tau) d \tau
\end{aligned}
$$


with

$$
B_{21}(\tau)=\int_{0}^{\infty}\left\langle i\left(\tau-\left(\xi^{3}-\rho \xi\right)\right)+\xi^{2}\right\rangle^{2 b}\langle\xi\rangle^{2 s}|(1+\omega(\tau))(1-\Theta(\xi, \tau))|^{2} \frac{\alpha_{\mu}^{2}(\tau) \phi_{2}(\mu(\tau))}{\left(\alpha_{\mu}^{2}(\tau)+\left(\xi-\beta_{\mu}(\tau)\right)^{2}\right)^{2}} d \xi
$$

Claim. If $b<1 / 2-s / 3$, then as $\tau \rightarrow \infty$,

$$
B_{21}(\tau) \sim \tau^{(6 b+2 s-1) / 3} .
$$

To see the claim is valid, note that in fact

$$
B_{21}(\tau)=\int_{\delta|\tau|^{1 / 3}}^{\infty}\left\langle i\left(\tau-\left(\xi^{3}-\rho \xi\right)\right)+\xi^{2}\right\rangle^{2 b}\langle\xi\rangle^{2 s} \frac{\alpha_{\mu}^{2}(\tau) \phi_{2}(\mu(\tau))}{\left(\alpha_{\mu}^{2}(\tau)+\left(\xi-\beta_{\mu}(\tau)\right)^{2}\right)^{2}}(1+\omega(\tau))^{2}(1-\Theta(\xi, \tau))^{2} d \xi
$$

since $\Theta(\xi, \tau)=1$ when $\xi<\delta|\tau|^{\frac{1}{3}}$, where $\delta>0$ is fixed, but arbitrary for the nonce. Let $\xi=\eta(\zeta)$ be the real solution of the equation

$$
\xi^{3}-\xi=\zeta, \quad 0 \leqslant \zeta<\infty, \frac{1}{\sqrt{3}} \leqslant \xi<\infty,
$$

that connects continuously to the unique real root as $\zeta$ becomes large. Note that

$$
\eta(\zeta) \sim \zeta^{1 / 3} \text { as } \zeta \rightarrow \infty
$$

For large $\tau$, it is also the case that

$$
\mu(\tau) \sim \tau^{1 / 3}, \quad\left|\alpha_{\mu}(\tau)\right| \sim \frac{\sqrt{3}}{2} \tau^{1 / 3}, \quad\left|\beta_{\mu}(\tau)\right| \sim \frac{1}{2} \tau^{1 / 3} .
$$

Thus, for $\tau>0$ large enough,

$$
\begin{aligned}
B_{21}(\tau) \leqslant & C \int_{\delta^{3} \tau}^{\infty} \frac{\tau^{2 / 3}\langle\tau-\zeta\rangle^{2 b}}{\left(1+3 \tau^{2 / 3}+\left(2 \eta(\zeta)-\tau^{1 / 3}\right)^{2}\right)^{2}}\langle\zeta\rangle^{2 s / 3} \frac{1}{3 \eta^{2}(\zeta)-1} d \zeta \\
= & C \int_{\delta^{3} \tau}^{2 \tau} \frac{\tau^{2 / 3}\langle\tau-\zeta\rangle^{2 b}}{\left(1+3 \tau^{2 / 3}+\left(2 \eta(\zeta)-\tau^{1 / 3}\right)^{2}\right)^{2}}\langle\zeta\rangle^{2 s / 3} \frac{1}{3 \eta^{2}(\zeta)-1} d \zeta \\
& +C \int_{2 \tau}^{\infty} \frac{\tau^{2 / 3}\langle\tau-\zeta\rangle^{2 b}}{\left(1+3 \tau^{2 / 3}+\left(2 \eta(\zeta)-\tau^{1 / 3}\right)^{2}\right)^{2}}\langle\zeta\rangle^{2 s / 3} \frac{1}{3 \eta^{2}(\zeta)-1} d \zeta \\
:= & G_{21-1}(\tau)+G_{21-2}(\tau) .
\end{aligned}
$$

Continuing this sequence of inequalities, note further that

$$
\begin{aligned}
G_{21-1}(\tau) & \leqslant C \frac{\tau^{2 / 3}}{\left(1+\tau^{2 / 3}\right)^{2}} \int_{\delta^{3} \tau}^{2 \tau} \frac{\langle\tau-\zeta\rangle^{2 b}}{(1+|\zeta|)^{-2 s / 3}} \frac{1}{3 \eta^{2}(\zeta)-1} d \zeta \\
& \leqslant C \frac{\tau^{2 / 3}(1+\tau)^{2 b}}{\left(1+\tau^{2 / 3}\right)^{2}} \int_{\delta^{3} \tau}^{2 \tau} \frac{1}{(1+\zeta)^{(2-2 s) / 3}} d \zeta \\
& \leqslant C \tau^{(6 b+2 s-1) / 3}
\end{aligned}
$$

and 


$$
\begin{aligned}
G_{21-2}(\tau) & \leqslant C \tau^{2 / 3} \int_{2 \tau}^{\infty} \frac{\langle\tau-\zeta\rangle^{2 b}}{\left(1+\tau^{2 / 3}+\zeta^{2 / 3}\right)^{2}(1+\zeta)^{-2 s / 3} \zeta^{2 / 3}} d \zeta \\
& \leqslant C \tau^{2 / 3} \int_{2 \tau}^{\infty} \frac{\zeta^{2 b}}{\zeta^{2(1-s / 3)}} d \zeta \\
& \leqslant C \tau^{\frac{6 b+2 s-1}{3}}
\end{aligned}
$$

if $b<1 / 2-s / 3$. The claim is thereby established.

As a consequence, the following estimate emerges. For given $s \leqslant 0$ and $b<1 / 2-s / 3$, there exists a constant $C$ such that

$$
\begin{gathered}
\int_{\frac{2}{3 \sqrt{3}}}^{\infty} \phi_{2}(\mu(\tau))\left|\int_{0}^{\infty} h(s) e^{-i s \tau} d s\right|^{2} B_{21}(\tau) d \tau \leqslant C \int_{\frac{2}{3 \sqrt{3}}}^{\infty} \phi_{2}^{2}(\mu(\tau)) \tau^{2(3 b+s-1 / 2) / 3}\left|\int_{0}^{\infty} h(s) e^{-i s \tau} d s\right|^{2} d \tau \\
\leqslant C\|h\|_{H^{(3 b+s-1 / 2) / 3}\left(\Re^{+}\right)}^{2}
\end{gathered}
$$

for any $h \in H^{(3 b+s-1 / 2) / 3}\left(\Re^{+}\right)$. The proof of Proposition 5.1 is complete.

Next, attention is given to the term

$$
\int_{-\infty}^{\infty} \int_{-\infty}^{\infty}\left\langle i\left(\tau-\left(\xi^{3}-\rho \xi\right)\right)+\xi^{2}\right\rangle^{2 b}\langle\xi\rangle^{2 s}\left|\hat{I}_{22}(\xi, \tau)\right|^{2} d \xi d \tau
$$

Proposition A.2. Let $s$ and $b$ be given satisfying $s>-\frac{3}{2}$ and $0 \leqslant b<\frac{5}{6}-\frac{s}{3}$. Then there is a constant $C$ such that

$$
\int_{-\infty}^{\infty} \int_{-\infty}^{\infty}\left\langle i\left(\tau-\left(\xi^{3}-\rho \xi\right)\right)+\xi^{2}\right\rangle^{2 b}\langle\xi\rangle^{2 s}\left|\hat{I}_{22}(\xi, \tau)\right|^{2} d \xi d \tau<C\|h\|_{H^{\left(3 b+s-\frac{1}{2}\right) / 3}\left(\Re^{+}\right)}^{2}
$$

for any $h \in H^{\left(3 b+s-\frac{1}{2}\right) / 3}\left(\mathfrak{R}^{+}\right)$.

Proof. As before, we only explicitly estimate one term in $\mathcal{F}_{t}\left[\int_{0}^{\infty} E(x, t) \cos (x \eta) d x\right]$, say the term

$$
A_{11}(\xi, \tau)=K_{11}(\xi, \tau) \phi_{2}(\mu(\tau)) \hat{h}(\tau) .
$$

Notice that $A_{11}(-\xi, \tau)=A_{21}(\xi, \tau)$. Hence, we may consider only the case where $\xi \geqslant 0$. Denote by $q_{2}$ the quantity

$$
q_{2}(\xi, \tau)=\frac{1}{\alpha_{\mu}^{2}(\tau)+\left(\xi+\beta_{\mu}(\tau)\right)^{2}}
$$

and, for $\xi \geqslant 1$, let $D_{2}$ be given by

$$
D_{2}(\xi, \tau)=2 \int_{-\infty}^{\infty} \frac{\eta^{2} \Theta(\eta, \tau)}{\xi\left(\xi^{2}-\eta^{2}\right)} q_{2}(\eta, \tau) d \eta+(1+\omega(\tau)) \int_{-\infty}^{\infty} \frac{\eta^{2}(1-\Theta(\eta, \tau))}{\xi\left(\xi^{2}-\eta^{2}\right)} q_{2}(\eta, \tau) d \eta
$$

for $\xi \geqslant \delta_{1} \mu(\tau)$ and

$$
D_{2}(\xi, \tau)=2 \int_{-\infty}^{\infty} \frac{\xi \Theta(\eta, \tau)}{\xi^{2}-\eta^{2}} q_{2}(\eta, \tau) d \eta+(1+\omega(\tau)) \int_{-\infty}^{\infty} \frac{\xi(1-\Theta(\eta, \tau))}{\xi\left(\xi^{2}-\eta^{2}\right)} q_{2}(\eta, \tau) d \eta
$$

for $0 \leqslant \xi \leqslant \delta_{1} \mu(\tau)$, where $\delta_{1}>0$ is a small positive constant. The relevance of these functions will become clear presently. First, note that

$$
A_{11}(\xi, \tau)=q_{2}(\xi, \tau) \phi_{2}(\mu(\tau)) \hat{h}(\tau)|\alpha(\mu(\tau))| .
$$


As for $D_{2}$, changing variables in the integrals of its definition shows it to have the form

$$
\begin{aligned}
D_{2}(\xi, \tau)= & 2 \int_{0}^{\infty} \frac{\eta^{2}}{\xi\left(\xi^{2}-\eta^{2}\right)} \Theta(\eta, \tau) q_{2}(\eta, \tau) d \eta+(1+\omega(\tau)) \int_{0}^{\infty} \frac{\eta^{2}}{\xi\left(\xi^{2}-\eta^{2}\right)}(1-\Theta(\eta, \tau)) q_{2}(\eta, \tau) d \eta \\
= & \frac{2}{\mu^{2}(\tau)} \int_{0}^{a_{0}} \frac{\eta^{2}}{y\left(y^{2}-\eta^{2}\right)} \Theta(\mu(\tau) \eta, \tau) p_{2}(\eta, \tau) d \eta \\
& +\frac{1+\omega(\tau)}{\mu^{2}(\tau)} \int_{a_{1}}^{\infty} \frac{\eta^{2}}{y\left(y^{2}-\eta^{2}\right)}(1-\Theta(\mu(\tau) \eta, \tau)) p_{2}(\eta, \tau) d \eta \\
:= & D_{21}(y, \tau)+D_{22}(y, \tau)
\end{aligned}
$$

where

$$
a_{0}=\frac{\delta|\tau|^{1 / 3}+1}{\mu(\tau)}, \quad a_{1}=\frac{\delta|\tau|^{1 / 3}}{\mu(\tau)}, \quad y=\frac{\xi}{\mu(\tau)}, \quad p_{2}(\eta, \tau)=\left(\frac{\alpha_{\mu}^{2}(\tau)}{\mu(\tau)^{2}}+\left(\eta+\frac{\beta_{\mu}(\tau)}{\mu(\tau)}\right)^{2}\right)^{-1} .
$$

We have similar definitions for $0 \leqslant y \leqslant \delta_{1}$. Remark that $a_{0}$ is bounded independently of $\tau$ and so for $y$ large enough, $y^{2}-\eta^{2}$ is bounded below for $\eta \in\left[0, a_{0}\right]$. Thus,

$$
\begin{aligned}
D_{21}(y, \tau) & =\frac{2}{y^{3} \mu^{2}(\tau)} \int_{0}^{a_{0}} \frac{\eta^{2}}{1-(\eta / y)^{2}} \Theta(\mu(\tau) \eta, \tau) p_{2}(\eta, \tau) d \eta \\
& :=\frac{1}{y^{3} \mu^{2}(\tau)} D_{21,2}(\tau, y)
\end{aligned}
$$

with

$$
\left|D_{21,2}(\tau, y)\right|<C \text { for all } \tau \text { and } y .
$$

Turning to $D_{22}$, note that $\Theta(\mu(\tau) \eta, \tau)=0$ for $\eta \geqslant a_{1}$, so

$$
\begin{aligned}
\int_{a_{1}}^{\infty} \frac{\eta^{2}}{y\left(y^{2}-\eta^{2}\right)}(1-\Theta(\mu(\tau) \eta, \tau)) p_{2}(\eta, \tau) d \eta & =\int_{a_{1}}^{\infty} \frac{\eta^{2}}{y\left(y^{2}-\eta^{2}\right)} p_{2}(\eta, \tau) d \eta \\
& =\frac{1}{y^{2}} \int_{\frac{a_{1}}{y}}^{\infty} \frac{1}{1-z^{2}} z^{2} y^{2} p_{2}(z y, \tau) d z \\
& =\frac{1}{y^{2}} \int_{\frac{a_{1}}{y}}^{\infty} \frac{1}{1-z^{2}}\left(z^{2} y^{2} p(z y, \tau)-\frac{1}{4}\right) d z+\frac{1}{4 y^{2}} \int_{\frac{a_{1}}{y}}^{\infty} \frac{1}{1-z^{2}} d z \\
& :=\frac{1}{y^{2}}\left(D_{22,1}(y, \tau)+D_{22,2}(y, \tau)\right)
\end{aligned}
$$

Of course,

$$
D_{22,2}(y, \tau)=\frac{1}{4} \int_{\frac{a_{1}}{y}}^{\infty} \frac{1}{1-z^{2}} d z=-\int_{0}^{\frac{a_{1}}{y}} \frac{1}{1-\eta^{2}} d \eta
$$


since

$$
\int_{0}^{\infty} \frac{1}{1-\eta^{2}} d \eta=0
$$

as a principal-value integral. It is therefore clear that

$$
\left|D_{22,2}(y, \tau)\right| \leqslant \frac{C}{y}
$$

for some constant $C$ independent of $\tau$ when $y$ is large. As for $D_{22,1}(y, \tau)$, note that

$$
\begin{aligned}
\eta^{2} y^{2} p(\eta y, \tau)-1 & =\frac{1}{y}\left(2 \eta-\frac{\alpha_{\mu}^{2}(\tau)+\beta_{\mu}^{2}(\tau)}{\mu(\tau)^{2}} \frac{1}{y}\right)\left(\left(\frac{\alpha_{\mu}(\tau)}{\mu(\tau) y}\right)^{2}+\left(\eta+\frac{\beta_{\mu}(\tau)}{\mu(\tau) y}\right)^{2}\right)^{-1} \\
& :=\frac{1}{y} p^{*}(\eta, y, \tau) .
\end{aligned}
$$

Rewrite $D_{22,1}(y, \tau)$ as

$$
D_{22,1}(y, \tau)=\frac{1}{y}\left(\int_{a_{1} / y}^{1 / 2}+\int_{1 / 2}^{2}+\int_{2}^{\infty}\right) \frac{p^{*}(\eta, y, \tau)}{1-\eta^{2}} d \eta
$$

to obtain

$$
\left|\left(\int_{1 / 2}^{2}+\int_{2}^{\infty}\right) \frac{p^{*}(\eta, y, \tau)}{1-\eta^{2}} d \eta\right| \leqslant C
$$

and

$$
\left|\int_{a_{1} / y}^{1 / 2} \frac{p^{*}(\eta, y, \tau)}{1-\eta^{2}} d \eta\right| \leqslant C(1+\ln y)
$$

where $C$ is independent of $\tau$ and $y$ for $\mu(\tau) \geqslant 3$ and $y$ large. Thus, if $y>y_{0}$, then

$$
\left|D_{2}(\mu(\tau) y, \tau)\right| \leqslant \frac{C}{y^{3}}(1+\ln y) \frac{1}{\mu^{2}(\tau)}
$$

where $C$ is independent of $\tau$ and $y$. The following calculation shows the relevance of $D_{2}$;

$$
\begin{aligned}
& \int_{0}^{\infty} \int_{0}^{\infty}\left\langle i\left(\tau-\left(\xi^{3}-\rho \xi\right)\right)+\xi^{2}\right\rangle^{2 b}\langle\xi\rangle^{2 s}\left|\int_{-\infty}^{\infty} \frac{1}{\xi-\eta} A_{11}(\eta, \tau)(2 \Theta(\eta, \tau)+(1-\Theta(\eta, \tau))(1+\omega(\tau))) d \eta\right|^{2} d \xi d \tau \\
& =\int_{0}^{\infty} \frac{1}{\pi^{2}} \phi_{2}^{2}(\mu(\tau))|\hat{h}|^{2}(\tau) \alpha_{\mu}^{2}(\tau) \int_{0}^{\infty}\left\langle i\left(\tau-\left(\xi^{3}-\rho \xi\right)\right)+\xi^{2}\right\rangle^{2 b}\langle\xi\rangle^{2 s} \\
& \quad \times\left|\int_{-\infty}^{\infty} \frac{1}{\xi-\eta} q_{2}(\eta, \tau)(2 \Theta(\eta, \tau)+(1-\Theta(\eta, \tau))(1+\omega(\tau))) d \eta\right|^{2} d \xi d \tau \\
& =\int_{0}^{\infty} \frac{1}{\pi^{2}} \phi_{2}^{2}(\mu(\tau))|\hat{h}|^{2}(\tau) \alpha_{\mu}^{2}(\tau) \int_{0}^{\infty}\left\langle i\left(\tau-\left(\xi^{3}-\rho \xi\right)\right)+\xi^{2}\right\rangle^{2 b}\langle\xi\rangle^{2 s}\left|D_{2}(\xi, \tau)\right|^{2} d \xi d \tau .
\end{aligned}
$$

Appropriate bounds on $D_{2}$ yield bounds on the left-hand side of the last formula. Consider the quantity 


$$
\begin{aligned}
E_{2}(\tau) & :=\alpha_{\mu}^{2}(\tau) \int_{0}^{\infty}\left\langle i\left(\tau-\left(\xi^{3}-\rho \xi\right)\right)+\xi^{2}\right\rangle^{2 b}\langle\xi\rangle^{-2 s}\left|D_{2}(\xi, \tau)\right|^{2} d \xi \\
& =\alpha_{\mu}^{2}(\tau)\left(\int_{0}^{\delta_{1} \mu(\tau)}+\int_{\delta_{1} \mu(\tau)}^{y_{0} \mu(\tau)}+\int_{y_{0} \mu(\tau)}^{\infty}\right)\left\langle i\left(\tau-\left(\xi^{3}-\rho \xi\right)\right)+\xi^{2}\right\rangle^{2 b}\langle\xi\rangle^{2 s} D_{2}^{2}(\xi, \tau) d \xi \\
& :=E_{21}(\tau)+E_{22}(\tau)+E_{23}(\tau),
\end{aligned}
$$

where $\delta_{1}$ is again a small positive constant. By our choice of $\omega(\tau)$,

$$
\begin{aligned}
\left|E_{23}(\tau)\right| & \leqslant C \tau^{2 / 3} \int_{y_{0} \mu(\tau)}^{\infty} \xi^{6 b+2 s-6} d \xi \\
& \leqslant C \tau^{2 / 3} \mu(\tau)^{6 b+2 s-3} \int_{y_{0}}^{\infty} \xi^{6 b+2 s-6} d \xi \\
& \leqslant C \tau^{2 b+2 s / 3-1 / 3}
\end{aligned}
$$

for large $\tau$, provided $6 b+2 s-6<1$, which is to say, $b<(-2 s+5) / 6$. For $\delta_{1} \leqslant y \leqslant y_{0}$, say,

$$
\left|D_{2}\right| \leqslant \frac{C}{\mu^{2}(\tau)}\left(\left|\int_{0}^{a_{0}} \frac{1}{y-\eta} \Theta(\mu(\tau) \eta, \tau) p_{2}(\eta, \tau) d \eta+(1+\omega(\tau)) \int_{a_{1}}^{\infty} \frac{1}{y-\eta}(1-\Theta(\mu(\tau) \eta, \tau)) p_{2}(\eta, \tau) d \eta\right|\right) .
$$

Note that if $\delta_{1} \leqslant y \leqslant a_{0}$, then

$$
\begin{aligned}
\left|\int_{0}^{a_{0}} \frac{1}{y-\eta} \Theta(\mu(\tau) \eta, \tau) p_{2}(\eta, \tau) d \eta\right| \leqslant & \left|\int_{0}^{a_{0}} \frac{\Theta(\mu(\tau) \eta, \tau)}{y-\eta}\left(p_{2}(\eta, \tau)-p_{2}(y, \tau)\right) d \eta\right| \\
& +\left|p_{2}(y, \tau) \int_{0}^{a_{0}} \frac{\Theta(\mu(\tau) \eta, \tau)}{y-\eta} d \eta\right| \leqslant C .
\end{aligned}
$$

The same bound is valid if $a_{1} \leqslant y \leqslant y_{0}$; thus

$$
\left|D_{2}\right| \leqslant \frac{C}{\mu^{2}(\tau)}
$$

and

$$
\begin{aligned}
\left|E_{22}(\tau)\right| & \leqslant C \tau^{-2 / 3} \int_{\delta_{1} \mu(\tau)}^{y_{0} \mu(\tau)}\left\langle i\left(\tau-\left(\xi^{3}-\rho \xi\right)\right)+\xi^{2}\right\rangle^{2 b}\langle\xi\rangle^{2 s} d \xi \\
& \leqslant C \tau^{-2 / 3}\left(\int_{\delta_{1} \mu(\tau)}^{\tau^{1 / 3} / 2}+\int_{\tau^{1 / 3} / 2}^{2 \tau^{1 / 3}}+\int_{2 \tau^{1 / 3}}^{y_{0} \mu(\tau)}\right)\left\langle i\left(\tau-\left(\xi^{3}-\rho \xi\right)\right)+\xi^{2}\right\rangle^{2 b}\langle\xi\rangle^{2 s} d \xi \\
& \leqslant C \tau^{-2 / 3}\left(\tau^{2 b} \int_{\delta_{1} \mu(\tau)}^{\tau^{1 / 3} / 2}\langle\xi\rangle^{2 s} d \xi+\int_{\tau^{1 / 3} / 2}^{2 \tau^{1 / 3}}\left\langle i\left(\tau-\left(\xi^{3}-\rho \xi\right)\right)+\xi^{2}\right\rangle^{2 b} \xi^{2 s} d \xi+\int_{2 \tau^{1 / 3}}^{y_{0} \mu(\tau)} \xi^{6 b+2 s} d \xi\right) \\
& \leqslant C \tau^{-2 / 3}\left(\tau^{2 b+(1+2 s) / 3}+\int_{1 / 2}^{2}(1+\tau|\xi-1|)^{2 b} \tau^{(1+2 s) / 3} \xi^{2 s} d \xi+\tau^{(6 b+2 s+1) / 3}\right)
\end{aligned}
$$




$$
\begin{aligned}
& \leqslant C \tau^{-2 / 3}\left(\tau^{(6 b+2 s+1) / 3}+\tau^{2 b+(1+6 s) / 3}\right) \\
& \leqslant C \tau^{(6 b+2 s-1) / 3}
\end{aligned}
$$

since $b \geqslant 0$. If $0 \leqslant y \leqslant \frac{\xi}{\mu(\tau)} \leqslant \delta_{1}$ in the decomposition $D_{2}=D_{21}+D_{22}$, then

$$
\left|D_{22}\right| \leqslant \frac{C}{\mu^{2}(\tau)}\left|\int_{a_{1}}^{\infty} \frac{2 y}{y^{2}-\eta^{2}}(1-\Theta(\mu(\tau) \eta, \tau)) p_{2}(\eta, \tau) d \eta\right| \leqslant C \frac{|y|}{\mu^{2}(\tau)}
$$

and

$$
\begin{aligned}
D_{21}= & \frac{1}{\mu^{2}(\tau)}\left(\int_{0}^{a_{0}} \frac{1}{y-\eta}\left(p_{2}(\eta, \tau)-p_{2}(y, \tau)\right) \Theta(\mu(\tau) \eta, \tau) d \eta\right. \\
& +\int_{0}^{a_{0}} \frac{1}{y+\eta}\left(p_{2}(\eta, \tau)-p_{2}(-y, \tau)\right) \Theta(\mu(\tau) \eta, \tau) d \eta \\
& \left.+\int_{0}^{a_{0}}\left(\frac{1}{y-\eta} p_{2}(y, \tau)+\frac{1}{y+\eta} p_{2}(-y, \tau)\right) \Theta(\mu(\tau) \eta, \tau) d \eta\right) \\
:= & \frac{1}{\mu^{2}(\tau)}\left(D_{21-1}+D_{21-2}+D_{21-3}\right) .
\end{aligned}
$$

Recall that $p_{2}(\eta, \tau)=\left(v^{2}(\tau)+(\eta+w(\tau))^{2}\right)^{-1}$ with

$$
v(\tau)=\frac{\alpha_{\mu}(\tau)}{\mu(\tau)}, \quad w(\tau)=\frac{\beta_{\mu}(\tau)}{\mu(\tau)},
$$

so that

$$
\begin{aligned}
D_{21-1}(y, \tau)+D_{21-2}(y, \tau)= & \int_{0}^{a_{0}} \frac{\Theta(\mu(\tau) \eta, \tau)}{v^{2}(\tau)+(\eta+w(\tau))^{2}}\left(\frac{y+\eta+2 w(\tau)}{v^{2}(\tau)+(y+w(\tau))^{2}}-\frac{\eta-y+2 w(\tau)}{v^{2}(\tau)+(-y+w(\tau))^{2}}\right) d \eta \\
= & \int_{0}^{a_{0}} \frac{\Theta(\mu(\tau) \eta, \tau)}{v^{2}(\tau)+(\eta-w(\tau))^{2}}\left[\frac{-4 y w(\tau)(\eta+2 w(\tau))}{\left(v^{2}(\tau)+(y-w(\tau))^{2}\right)\left(v^{2}(\tau)+(y+w(\tau))^{2}\right)}\right. \\
& \left.+y\left(\frac{1}{v^{2}(\tau)+(y-w(\tau))^{2}}+\frac{1}{v^{2}(\tau)+(y+w(\tau))^{2}}\right)\right] d \eta .
\end{aligned}
$$

It thus transpires that

$$
\left|D_{21-1}+D_{21-2}\right| \leqslant C|y| \text {. }
$$

We also note that

$$
\begin{aligned}
D_{21-3}= & p_{2}(y, \tau) \int_{0}^{a_{0}} \frac{1}{y-\eta} \Theta(\mu(\tau) \eta, \tau) d \eta+p_{2}(-y, \tau) \int_{0}^{a_{0}} \frac{1}{y+\eta} \Theta(\mu(\tau) \eta, \tau) d \eta \\
= & p_{2}(y, \tau)\left(\int_{0}^{a_{1}} \frac{1}{y-\eta} d \eta+\int_{a_{1}}^{a_{0}} \frac{1}{y-\eta} \Theta(\mu(\tau) \eta, \tau) d \eta\right) \\
& +p_{2}(-y, \tau)\left(\int_{0}^{a_{1}} \frac{1}{y+\eta} d \eta+\int_{a_{1}}^{a_{0}} \frac{1}{y+\eta} \Theta(\mu(\tau) \eta, \tau) d \eta\right)
\end{aligned}
$$




$$
\begin{aligned}
= & p_{2}(y, \tau)\left(-\ln \left|a_{1}-y\right|+\ln |y|\right)+p_{2}(-y, \tau)\left(\ln \left|a_{1}+y\right|-\ln |y|\right) \\
& +p_{2}(y, \tau) \int_{a_{1}}^{a_{0}} \frac{1}{\eta} \frac{\Theta(\mu(\tau) \eta, \tau) d \eta}{y / \eta-1}+p_{2}(-y, \tau) \int_{a_{1}}^{a_{0}} \frac{1}{\eta} \frac{\Theta(\mu(\tau) \eta, \tau) d \eta}{y / \eta+1} \\
= & \left(-p_{2}(y, \tau)+p_{2}(-y, \tau)\right)\left(\ln \left|a_{1}\right|-\ln |y|\right)+p_{2}(y, \tau)\left(-\ln \left|1-\frac{y}{a_{1}}\right|\right) \\
& +p_{2}(-y, \tau) \ln \left(1+\frac{y}{a_{1}}\right)+p_{2}(y, \tau) \int_{a_{1}}^{a_{0}} \frac{1}{\eta}\left(-1+\frac{y / \eta}{y / \eta-1}\right) \Theta(\mu(\tau) \eta, \tau) d \eta \\
& +p_{2}(-y, \tau) \int_{a_{1}}^{a_{0}} \frac{1}{\eta}\left(1-\frac{y / \eta}{y / \eta+1}\right) \Theta(\mu(\tau) \eta, \tau) d \eta \\
= & \left(-p_{2}(y, \tau)+p_{2}(-y, \tau)\right)\left(\ln \left|a_{1}\right|-\ln |y|+\int_{a_{1}}^{a_{0}} \frac{1}{\eta} \Theta(\mu(\tau) \eta, \tau) d \eta\right) \\
& +p_{2}(y, \tau)\left(-\ln \left(1-\frac{y}{a_{1}}\right)+\int_{a_{1}}^{a_{0}} \frac{y}{(y-\eta) \eta} \Theta(\mu(\tau) \eta, \tau) d \eta\right) \\
& +p_{2}(-y, \tau)\left(\ln \left(1+\frac{y}{a_{1}}\right)-\int_{a_{1}}^{y} \frac{y+\eta) \eta}{(y)}(\mu(\tau) \eta, \tau) d \eta\right) .
\end{aligned}
$$

As a consequence,

$$
\left|D_{21-3}\right| \leqslant C|y|(\ln |y|+1)
$$

and

$$
\left|D_{21}\right| \leqslant \frac{C|y|(\ln |y|+1)}{\mu^{2}(\tau)},
$$

which implies that

$$
\left|D_{2}\right| \leqslant \frac{C|y|(\ln |y|+1)}{\mu^{2}(\tau)} .
$$

Thus, it is apparent that

$$
\begin{aligned}
\left|E_{21}\right| & \leqslant C \tau^{2 / 3} \int_{0}^{\delta_{1} \mu(\tau)}(1+|\tau|)^{2 b}\langle\xi\rangle^{2 s} \frac{\xi^{2}}{\tau^{2}}\left(1+\ln \left|\frac{\xi}{\mu(\tau)}\right|\right) d \xi \\
& \leqslant C \tau^{-4 / 3}(1+|\tau|)^{2 b} \int_{0}^{\delta_{1} \mu(\tau)}\langle\xi\rangle^{2 s}\left(1+\ln \left|\frac{\xi}{\mu(\tau)}\right|\right) d \xi \\
& \leqslant C \tau^{-4 / 3}(1+|\tau|)^{2 b} \int_{0}^{\delta_{1}}(1+|\mu(\tau)||\xi|)^{2 s} \mu^{3}(\tau)(1+\ln |\xi|) \xi^{2} d \xi \\
& \leqslant C \tau^{-\frac{1}{3}+2 b+\frac{2 s}{3}} \int_{0}^{\delta_{1}} \xi^{2+2 s}(1+\ln |\xi|) d \xi \\
& \leqslant C \tau^{2 b-(1-2 s) / 3}
\end{aligned}
$$


if $2+2 s>-1$. Combining these estimates, there obtains

$$
\left|E_{2}(\tau)\right| \leqslant C \tau^{2 b-(1-2 s) / 3}
$$

if $s>-3 / 2$ and $0<b<\frac{1}{2}-\frac{s}{3}$. This in turn implies that

$$
\begin{aligned}
& \int_{-\infty}^{\infty} \int_{0}^{\infty}\left\langle i\left(\tau-\left(\xi^{3}-\rho \xi\right)\right)+\xi^{2}\right\rangle^{2 b}\langle\xi\rangle^{2 s} \\
& \quad \times\left|\int_{-\infty}^{\infty} \frac{1}{\xi-\eta} A_{21}(\eta, \tau) \times(2 \Theta(\eta, \tau)+(1-\Theta(\eta, \tau))(1+\omega(\tau))) d \eta\right|^{2} d \xi d \tau \\
& \leqslant C \int_{0}^{\infty} \tau^{2 b-(1-2 s) / 3}\left|\int_{0}^{\infty} h(s) e^{-i s \tau} d s\right|^{2} d \tau \\
& \leqslant C\|h\|_{H^{b+\frac{s}{3}-\frac{1}{6}}}^{2}
\end{aligned}
$$

Similar estimates for other terms yield, in sum,

$$
\int_{-\infty}^{\infty} \int_{-\infty}^{\infty}\left\langle i\left(\tau-\left(\xi^{3}-\rho \xi\right)\right)+\xi^{2}\right\rangle^{2 b}\langle\xi\rangle^{2 s}\left|\hat{I}_{22}(\xi, \tau)\right|^{2} d \xi d \tau \leqslant C\|h\|_{H^{b+\frac{s}{3}-\frac{1}{6}}}^{2}
$$

if $-\frac{3}{2}<s \leqslant 0$ and $0 \leqslant b<\frac{1}{2}-\frac{s}{3}$. This completes the proof of Proposition A.2.

Combining the last two propositions completes the proof of Proposition 2.8.

Proof of Proposition 2.9. As in the proof of Proposition 2.8, it suffices to establish the following two results.

Proposition A.3. Let $s \in \Re$ and $b \geqslant 0$ be given. There exists a constant $C$ such that

$$
\int_{-\infty}^{\infty} \int_{-\infty}^{\infty}\left\langle i\left(\tau-\left(\xi^{3}-\rho \xi\right)\right)+\xi^{2}\right\rangle^{2 b}\langle\xi\rangle^{2 s}\left|\hat{I}_{21}^{*}(\xi, \tau)\right|^{2} d \xi d \tau< \begin{cases}C\|h\|^{2} H^{\left(\frac{9 b+s-5 / 2}{9}\right.} & \text { if } s \geqslant-\frac{1}{2} \\ C\|h\|_{H^{2}}^{\frac{3 b-1}{3}} & \text { if } s \leqslant-\frac{1}{2}\end{cases}
$$

Proposition A.4. Let $s$ and $b$ be given satisfying $0 \leqslant b<\frac{5}{6}-\frac{s}{3}$. There exists a constant $C$ such that

$$
\int_{-\infty}^{\infty} \int_{-\infty}^{\infty}\left\langle i\left(\tau-\left(\xi^{3}-\rho \xi\right)\right)+\xi^{2}\right\rangle^{2 b}\langle\xi\rangle^{2 s}\left|\hat{I}_{22}^{*}(\xi, \tau)\right|^{2} d \xi d \tau<C\|h\|^{2}{ }_{H^{\frac{3 b+s-\frac{1}{2}}{3}}\left(\Re^{+}\right)}
$$

for any $h \in H^{\frac{3 b+s-\frac{1}{2}}{3}}\left(\Re^{+}\right)$.

We only present a proof of Proposition A.3. The proof of Proposition A.4 follows the same line as that of Proposition A.2 and may be safely omitted. As in the proof of Proposition A.1, detailed analysis is given for the term containing $K_{21}$; the estimates for the other terms are sufficiently similar that their proof do not require further elaboration. Suppose $\xi \geqslant 0$ and $\tau \geqslant 0$ in what follows. The other cases are entirely analogous. Define $A_{m 1}$ by

$$
A_{m 1}(\xi, \tau)=K_{m 1}(\xi, \tau) \phi_{2}(\mu(\tau)) \hat{h}(\tau), \quad m=1,2,3 .
$$


For given $s$ and $b \geqslant 0$, we have

$$
\begin{aligned}
& \int_{0}^{\infty} \int_{0}^{\infty}\left\langle i\left(\tau-\left(\xi^{3}-\rho \xi\right)\right)+\xi^{2}\right\rangle^{2 b}\langle\xi\rangle^{2 s}\left|A_{21}(\xi, \tau)(1+\omega(\tau)) \Theta(\xi, \tau)\right|^{2} d \xi d \tau \\
& \leqslant C \int_{-\infty}^{\infty} \phi_{2}(\mu(\tau))\left|\int_{0}^{\infty} h(s) e^{-i s \tau} d s\right|^{2} B_{21}^{*}(\tau) d \tau
\end{aligned}
$$

with

$$
\begin{aligned}
B_{21}^{*}(\tau) & =\int_{\sqrt{\rho}}^{\infty}\left\langle i\left(\tau-\left(\xi^{3}-\rho \xi\right)\right)+\xi^{2}\right\rangle^{2 b}\langle\xi\rangle^{2 s}|(1+\omega(\tau)) \Theta(\xi, \tau)|^{2} \frac{\alpha_{\mu}^{2}(\tau) \phi_{2}(\mu(\tau))}{\left(\alpha_{\mu}^{2}(\tau)+\left(\xi-\beta_{\mu}(\tau)\right)^{2}\right)^{2}} d \xi \\
& \leqslant C \int_{\sqrt{\rho}}^{\delta \tau^{1 / 3}}\left\langle i\left(\tau-\left(\xi^{3}-\rho \xi\right)\right)+\xi^{2}\right\rangle^{2 b}\langle\xi\rangle^{2 s} \frac{\alpha_{\mu}^{2}(\tau) \phi_{2}(\mu(\tau))}{\left(\alpha_{\mu}^{2}(\tau)+\left(\xi-\beta_{\mu}(\tau)\right)^{2}\right)^{2}} d \xi
\end{aligned}
$$

because of the properties of $\Theta$, and where $\delta>0$ is, as before, fixed, but arbitrary for the moment. Let $\xi=\eta(\zeta)$ be the unique real solution of the equation

$$
\begin{aligned}
& \xi^{3}-\rho \xi=\zeta, \quad 0 \leqslant \zeta<\infty, \sqrt{\rho} \leqslant \xi<\infty \\
& \eta(\zeta) \sim \zeta^{1 / 3} \text { as } \zeta \rightarrow \infty .
\end{aligned}
$$

For large $\tau$, note that $\mu(\tau) \sim \tau^{1 / 3}$. Thus, for $\tau>0$ large enough,

$$
\begin{aligned}
& B_{21}^{*}(\tau) \leqslant C \int_{0}^{\delta \tau^{1 / 3}} \frac{\left(3 \mu^{2}(\tau)-4\right)\langle\tau-\zeta\rangle^{2 b}}{\left(3 \mu^{2}(\tau)-4+(2 \eta(\zeta)-\mu(\tau))^{2}\right)^{2}}(1+|\zeta|)^{2 s / 3} \frac{1}{3 \eta^{2}(\zeta)-1} d \zeta \\
& \leqslant C \int_{0}^{\delta \tau^{1 / 3}} \frac{\tau^{\frac{2}{3}}\langle\tau-\zeta\rangle^{2 b}}{\left(1+3 \tau^{\frac{2}{3}}+\left(2 \eta(\zeta)-\tau^{\frac{1}{3}}\right)^{2}\right)^{2}}(1+|\zeta|)^{\frac{2 s}{3}} \frac{1}{3 \eta^{2}(\zeta)-1} d \zeta \\
& \leqslant C \frac{\tau^{\frac{2}{3}}}{\left(1+\tau^{\frac{2}{3}}\right)^{2}}\left(\int_{0}^{1}+\int_{1}^{\delta \tau^{\frac{1}{3}}}\right) \frac{\langle\tau-\zeta\rangle^{2 b}}{(1+|\zeta|)^{-\frac{2 s}{3}}} \frac{1}{3 \eta^{2}(\zeta)-1} d \zeta \\
& \leqslant C \frac{\tau^{\frac{2}{3}}}{\left(1+\tau^{\frac{2}{3}}\right)^{2}}\left((1+\tau)^{2 b} \int_{0}^{1} \frac{1}{\zeta^{\frac{2}{3}}} d \zeta+\int_{1}^{\delta \tau^{\frac{1}{3}}} \frac{\langle\tau-\zeta\rangle^{2 b}}{(1+\zeta)^{\frac{2-2 s}{3}}} d \zeta\right) \\
& \leqslant C \tau^{\frac{6 b-2}{3}}\left(1+\tau^{\frac{1+2 s}{9}}\right) \\
& \leqslant \begin{cases}C \tau^{\frac{18 b+2 s-5}{9}} & \text { if } s \geqslant-\frac{1}{2}, \\
C \tau^{\frac{6 b-2}{3}} & \text { if } s \leqslant-\frac{1}{2} .\end{cases}
\end{aligned}
$$

In consequence,

$$
\begin{aligned}
& \int_{0}^{\infty} \int_{0}^{\infty}\left\langle i\left(\tau-\left(\xi^{3}-\rho \xi\right)\right)+\xi^{2}\right\rangle^{2 b}\langle\xi\rangle^{2 s}\left|A_{21}(\xi, \tau)(1+\omega(\tau)) \Theta(\xi, \tau)\right|^{2} d \xi d \tau \\
& \quad \leqslant C \int_{0}^{\infty} \phi_{2}(\mu(\tau))\left|\int_{0}^{\infty} h(s) e^{-i s \tau} d s\right|^{2} B_{21}^{*}(\tau) d \tau
\end{aligned}
$$




$$
\begin{aligned}
& \leqslant C \int_{0}^{\infty} \phi_{2}(\mu(\tau))\left|\int_{0}^{\infty} h(s) e^{-i s \tau} d s\right|^{2} \begin{cases}C \tau^{\frac{18 b+2 s-5}{9}} d \tau & \text { if } s \geqslant-\frac{1}{2}, \\
C \tau^{\frac{6 b-2}{3}} d \tau & \text { if } s \leqslant-\frac{1}{2}\end{cases} \\
& = \begin{cases}C\|h\|^{2} H^{\frac{9 b+s-5 / 2}{9}} & \text { if } s \geqslant-\frac{1}{2}, \\
C\|h\|^{2}{ }_{H^{\frac{3 b-1}{3}}} & \text { if } s \leqslant-\frac{1}{2} .\end{cases}
\end{aligned}
$$

The proof is complete.

\section{Appendix B}

Proof of Lemma 2.6. Let $[\hat{I}(f)](k)$ denote the cosine transform of $[I(f)](t)$, which is to say,

$$
[\hat{I}(f)](k)=\int_{0}^{\infty} \cos k t \int_{-\infty}^{\infty} e^{i t \eta+\mu(\eta) t} f(\eta) d \eta d t .
$$

Since

$$
\|I\|_{L_{t}^{2}(\Re+)} \leqslant\|\hat{I}\|_{L_{k}^{2}(\Re)},
$$

it is sufficient to estimate only the terms

$$
\int_{0}^{\infty} \cos k t \int_{-\infty}^{\infty} e^{ \pm i k t+i t \eta+\mu(\eta) t} f(\eta) d \eta d t=\int_{-\infty}^{\infty} \frac{f(\eta)}{i(\eta \pm k)+\mu(\eta)} d \eta
$$

We only consider

$$
[I I(f)](k)=\int_{-\infty}^{\infty} \frac{f(\eta)}{i(\eta-k)+\mu(\eta)} d \eta
$$

as the other case follows by making the change of variables $\eta \rightarrow-\eta$. Rewrite $I I(k)$ as

$$
[I I(f)](k)=\left[I I_{1}(f)\right](k)-i\left[I_{2}(f)\right](k)
$$

with

$$
\left[I_{1}(f)\right](k)=\int_{-\infty}^{\infty} \frac{\mu(\eta)}{\mu^{2}(\eta)+(\eta-k)^{2}} f(\eta) d \eta
$$

and

$$
\left[I_{2}(f)\right](k)=\int_{-\infty}^{\infty} \frac{\eta-k}{\mu^{2}(\eta)+(\eta-k)^{2}} f(\eta) d \eta .
$$

To show that $\left\|I I_{1}(g)\right\|_{L_{2}(R)} \leqslant C\|f\|_{L_{2}(R)}$, it suffices to show that

$$
\sup _{\eta} \int_{-\infty}^{\infty}|K(\eta, k)| d k<C \quad \text { and } \quad \sup _{k} \int_{-\infty}^{\infty}|K(\eta, k)| d \eta<C
$$

where

$$
K(\eta, k)=\frac{\mu(\eta)}{\mu^{2}(\eta)+(\eta-k)^{2}} .
$$


It is straightforward to see that for any $\eta$,

$$
\int_{-\infty}^{\infty} \frac{|\mu(\eta)|}{\mu^{2}(\eta)+(\eta-k)^{2}} d k=\int_{-\infty}^{\infty} \frac{1}{v^{2}+1} d v=C
$$

Moreover, for $k$ bounded,

$$
\int_{-\infty}^{\infty}|K(\eta, k)| d \eta \leqslant C \int_{-\infty}^{\infty} \frac{\eta^{2 / 3}}{\eta^{4 / 3}+(\eta-k)^{2}} d \eta \leqslant C \int_{-\infty}^{\infty} \frac{\eta^{2 / 3}}{\eta^{4 / 3}+\eta^{2}} d \eta<\infty .
$$

For $k>0$ large,

$$
\begin{aligned}
\int_{-\infty}^{\infty} \frac{\eta^{2 / 3}}{\eta^{4 / 3}+(\eta-k)^{2}} d \eta & =\left(\int_{-\infty}^{0}+\int_{0}^{k / 2}+\int_{k / 2}^{2 k}+\int_{2 k}^{\infty}\right) \frac{\eta^{2 / 3}}{\eta^{4 / 3}+(\eta-k)^{2}} d \eta \\
\leqslant & C\left(\int_{-\infty}^{0} \frac{1}{\eta^{2 / 3}+\eta^{4 / 3}} d \eta+\int_{0}^{k / 2} \frac{\eta^{2 / 3}}{k^{2}+\eta^{4 / 3}} d \eta\right. \\
& \left.+\int_{k / 2}^{2 k} \frac{k^{2 / 3}}{(\eta-k)^{2}+k^{4 / 3}} d \eta+\int_{2 k}^{\infty} \frac{\eta^{2 / 3}}{\eta^{2}+\eta^{4 / 3}} d \eta\right) \leqslant C<\infty
\end{aligned}
$$

Thus, for any $f \in L^{2}(\Re)$,

$$
\left\|I I_{1}(f)\right\|_{L^{2}(\Re)} \leqslant C\|f\|_{L^{2}(\Re)} .
$$

Remark. Inequality (B.1) is also true if

$$
K(\eta)=\int_{-\infty}^{\infty} \frac{y_{0}+\eta^{2 / 3}}{(\eta-k)^{2}+\left(y_{0}+\eta^{2 / 3}\right)^{2}} d k
$$

To study $I I_{2}$, let $0<a \leqslant \infty$ be given and consider the function

$$
\Phi_{a}(z)=\int_{-a}^{a} \frac{f(t)}{i(t-z)-\mu(t)} d t
$$

It is analytic in the region $\operatorname{Im} z>0$ since the zeros of $i(t-z)+\mu(t)$ are $z=t+i \mu(t)$. In addition, if $f(t)$ is compactly supported, then

$$
\Phi_{a}(z) \sim O\left(\frac{1}{|z|}\right) \quad \text { as }|z| \rightarrow \infty
$$

and the implied constant is independent of $a$.

For given $r>0$ and $y_{0}>0$, let $C_{r}$ denote the closed curve in the $z$-plane which is taken along the line from $-r+i y_{0}$ to $r+i y_{0}$ with $y_{0}>0$ and then around the semicircle above it of radius $r$ centered at $i y_{0}$ in the counterclockwise direction. By Cauchy's theorem,

$$
\int_{C_{r}} \Phi_{a}(z)^{2} d z=0
$$

which, upon taking the limit as $r \rightarrow \infty$, yields

$$
\int_{-\infty}^{\infty} \Phi_{1}\left(x+i y_{0}\right)^{2} d x=0
$$


Since

$$
\Phi_{a}\left(x+i y_{0}\right)=U_{a}\left(x+i y_{0}\right)+i V_{a}\left(x+i y_{0}\right)
$$

with

$$
U_{a}\left(x+i y_{0}\right)=\int_{-a}^{a} \frac{y_{0}-\mu(t)}{(t-x)^{2}+\left(y_{0}-\mu(t)\right)^{2}} f(t) d t
$$

and

$$
V_{a}\left(x+i y_{0}\right)=-\int_{-a}^{a} \frac{t-x}{(t-x)^{2}+\left(y_{0}-\mu(t)\right)^{2}} f(t) d t,
$$

it follows that

$$
\int_{-\infty}^{\infty} U_{a}^{2}\left(x+i y_{0}\right) d x=\int_{-\infty}^{\infty} V_{a}^{2}\left(x+i y_{0}\right) d x
$$

and hence by Fubini's theorem,

$$
\begin{aligned}
\int_{-\infty}^{\infty} V_{a}^{2}\left(x+i y_{0}\right) d x & \leqslant \int_{-\infty}^{\infty}\left(\int_{-\infty}^{\infty} \frac{y_{0}-\mu(t)}{(t-x)^{2}+\left(y_{0}-\mu(t)\right)^{2}}|f(t)| d t\right)^{2} d x \\
& \leqslant C\|f\|_{L^{2}(\Re)}^{2} .
\end{aligned}
$$

Taking $y_{0} \rightarrow 0$ and applying Fatou's Lemma leads to the conclusion

$$
\int_{-\infty}^{\infty}\left(\int_{-a}^{a} \frac{t-x}{(t-x)^{2}+\mu^{2}(t)}|f(t)| d t\right)^{2} d x \leqslant C\|f\|_{L^{2}(\Re)}^{2} .
$$

Then, letting $a \rightarrow \infty$ and using Fatou's Lemma again yields

$$
\int_{-\infty}^{\infty}\left(\int_{-\infty}^{\infty} \frac{t-x}{(t-x)^{2}+\mu^{2}(t)}|f(t)| d t\right)^{2} d x \leqslant C\|f\|_{L^{2}(\Re)}^{2}
$$

for all compactly supported $f$. The proof is complete.

\section{References}

[1] D. Bekiranov, The initial-value problem for the generalized Burgers' equation, Differential Integral Equations 9 (1996) 1253-1265

[2] B. Boczar-Karakiewicz, J.L. Bona, Wave dominated shelves: a model of sand ridge formation by progressive infragravity waves, in: R.J. Knight, J.R. McLean (Eds.), Shelf Sands and Sandstones, in: Canadian Society of Petroleum Geologists Memoir, vol. 11, 1986, pp. 163179.

[3] J.L. Bona, P.J. Bryant, A mathematical model for long waves generated by wave makers in non-linear dispersive systems, Proc. Cambridge Philos. Soc. 73 (1973) 391.

[4] J.L. Bona, L. Luo, Generalized Korteweg-de Vries equation in a quarter plane, Contemp. Math. 221 (1999) 59-125.

[5] J.L. Bona, W.G. Pritchard, L.R. Scott, An evaluation of a model equation for water waves, Philos. Trans. Roy. Soc. London Ser. A 302 (1981) 457-510.

[6] J.L. Bona, L.R. Scott, Solutions of the Korteweg-de Vries equation in fractional order Sobolev spaces, Duke Math. J. 43 (1976) 87-99.

[7] J.L. Bona, R. Smith, The initial-value problem for the Korteweg-de Vries equation, Philos. Trans. Roy. Soc. London Ser. A 278 (1975) $555-601$.

[8] J.L. Bona, S. Sun, B.-Y. Zhang, A nonhomogeneous boundary-value problem for the Korteweg-de Vries equation in a quarter plane, Trans. Amer. Math. Soc. 354 (2001) 427-490.

[9] J.L. Bona, S. Sun, B.-Y. Zhang, Forced oscillations of a damped KdV equation in a quarter plane, Commun. Contemp. Math. 5 (2003) 369-400. 
[10] J.L. Bona, S. Sun, B.-Y. Zhang, A nonhomogeneous boundary value problem for the KdV equation posed on a finite domain, Commun. Partial Differential Equations 28 (2003) 1391-1436.

[11] J.L. Bona, S. Sun, B.-Y. Zhang, Conditional and unconditional well-posedness of non-linear evolution equations, Adv. Differential Equations 9 (2004) 241-265.

[12] J.L. Bona, S. Sun, B.-Y. Zhang, Boundary smoothing properties of the Korteweg-de Vries equation in a quarter plane and applications, Dynamics Partial Differential Equations 3 (2006) 1-70.

[13] J.L. Bona, R. Winther, The Korteweg-de Vries equation, posed in a quarter plane, SIAM J. Math. Anal. 14 (1983) $1056-1106$.

[14] J.L. Bona, R. Winther, Korteweg-de Vries equation in a quarter plane, continuous dependence results, Differential Integral Equations 2 (1989) $228-250$.

[15] J. Bourgain, Fourier transform restriction phenomena for certain lattice subsets and applications to non-linear evolution equations, part II: the KdV equation, Geom. Funct. Anal. 3 (1993) 209-262.

[16] J. Bourgain, Periodic Korteweg-de Vries equation with measures as initial data, Selecta Math. (N.S.) 3 (1997) $115-159$.

[17] A. Cohen, Solutions of the Korteweg-de Vries equation from irregular data, Duke Math. J. 45 (1978) 149-181.

[18] A. Cohen, Existence and regularity for solutions of the Korteweg-de Vries equation, Arch. Rat. Mech. Anal. 71 (1979) $143-175$.

[19] M. Christ, J. Colliander, T. Tao, Asymptotics, frequency modulation, and low regularity ill-posedness for canonical defocusing equations, Amer. J. Math. 125 (2003) 1235-1293.

[20] J.E. Colliander, C.E. Kenig, The generalized Korteweg-de Vries equation on the half line, Comm. Partial Differential Equations 27 (2002) 2187-2266.

[21] J.E. Colliander, M. Keel, G. Staffilani, H. Takaoka, T. Tao, Sharp global well-posedness for KdV and modified KdV on $R$ and $T$, J. Amer. Math. Soc. 16 (2003) 705-749.

[22] P. Constantin, J.-C. Saut, Local smoothing properties of dispersive equations, J. Amer. Math. Soc. 1 (1988) $413-446$.

[23] W. Craig, T. Kappeler, W.A. Strauss, Gain of regularity for equations of the Korteweg-de Vries type, Ann. Inst. H. Poincaré 9 (1992) $147-186$.

[24] D.B. Dix, The dissipation of non-linear dispersive waves: the case of asymptotically weak non-linearity, Comm. Partial Differential Equations 17 (1992) 1665-1693.

[25] D.B. Dix, Nonuniqueness and uniqueness in the initial-value problem for Burgers' equation, SIAM J. Math. Anal. 27 (1996) $708-724$.

[26] A.V. Faminskii, The Cauchy problem and the mixed problem in the half strip for equations of Korteweg-de Vries type, Dinamika Sploshn. Sredy 162 (1983) 152-158 (in Russian).

[27] A.V. Faminskii, A mixed problem in a semistrip for the Korteweg-de Vries equation and its generalizations, Dinamika Sploshn. Sredy 258 (1988) 54-94 (in Russian). English translation in Trans. Moscow Math. Soc. 51 (1989) 53-91.

[28] A.V. Faminskii, Mixed problems for the Korteweg-de Vries equation, Sb. Math. 190 (1999) 903-935.

[29] A.V. Faminskii, An initial boundary-value problem in a half-strip for the Korteweg-de Vries equation in fractional-order Sobolev spaces, Comm. Partial Differential Equations 29 (2004) 1653-1695.

[30] A.S. Fokas, A.R. Its, Soliton generation for initial-boundary value problems, Phys. Rev. Lett. 68 (1992) 3117-3120.

[31] A.S. Fokas, A.R. Its, An initial-boundary value problem for the Korteweg-de Vries equation, in: The Proceedings of the Conference: Solitons, Nonlinear Wave Equations and Computation, New Brunswick, NJ, 1992, Math. Comput. Simulation 37 (1994) $293-321$.

[32] A.S. Fokas, A.R. Its, Integrable equations on the half-infinite line. Solitons in science and engineering: theory and applications, Chaos Solitons Fractals 5 (1995) 2367-2376.

[33] A.S. Fokas, B. Pelloni, The solution of certain initial boundary-value problems for the linearized Korteweg-de Vries equation, Proc. Roy. Soc. London Ser. A 454 (1998) 645-657.

[34] J. Holmer, The initial-boundary value problem for the Korteweg-de Vries equation, Comm. Partial Differential Equations 31 (2006) 11511190.

[35] R.S. Johnson, A non-linear equation incorporating damping and dispersion, J. Fluid Mech. 42 (1970) 49-60.

[36] T. Kappeler, P. Topalov, Well-posedness of KDV on $H^{-1}(T)$, Duke Math. J. 135 (2006) 327-360.

[37] T. Kato, On the Korteweg-de Vries equation, Manuscripta Math. 29 (1979) 89-99.

[38] T. Kato, The Cauchy problem for the Korteweg-de Vries equation, in: Pitman Research Notes in Math., vol. 53, 1979 , pp. $293-307$.

[39] T. Kato, On the Cauchy problem for the (generalized) Korteweg-de Vries equations, in: Advances in Mathematics Supplementary Studies, in: Studies Appl. Math., vol. 8, 1983, pp. 93-128.

[40] C.E. Kenig, G. Ponce, L. Vega, On the (generalized) Korteweg-de Vries equation, Duke Math. J. 59 (1989) $585-610$.

[41] C.E. Kenig, G. Ponce, L. Vega, Well-posedness of the initial value problem for the KdV equation, J. Amer. Math. Soc. 4 (1991) $323-347$.

[42] C.E. Kenig, G. Ponce, L. Vega, Well-posedness and scattering results for the generalized Korteweg-de Vries equations via the contraction principle, Comm. Pure Appl. Math. 46 (1993) 527-620.

[43] C.E. Kenig, G. Ponce, L. Vega, The Cauchy problem for the Korteweg-de Vries equation in Sobolev spaces of negative indices, Duke Math. J. 71 (1993) 1-21.

[44] C.E. Kenig, G. Ponce, L. Vega, A bilinear estimate with applications to the KdV equation, J. Amer. Math. Soc. 9 (1996) $573-603$.

[45] C.E. Kenig, G. Ponce, L. Vega, On the ill-posedness of some canonical dispersive equations, Duke Math. J. 106 (2001) $617-633$.

[46] S.N. Kruzhkov, A.V. Faminskii, Generalized solutions of the Cauchy problem for the Korteweg-de Vries equation, Mat. Sb. (N.S.) 120 (1983) 396-425 (in Russian). English translation in Math. USSR Sb. 48 (1984) 391-421.

[47] L. Molinet, F. Ribaud, On the low regularity of the Korteweg-de Vries-Burgers equation, Int. Math. Res. Notices 37 (2002) $1979-2005$.

[48] D.L. Russell, B.-Y. Zhang, Smoothing properties of solutions of the Korteweg-de Vries equation on a periodic domain with point dissipation, J. Math. Anal. Appl. 190 (1995) 449-488.

[49] R.L. Sachs, Classical solutions of the Korteweg-de Vries equation for non-smooth initial data via inverse scattering, Comm. Partial Differential Equations 10 (1985) 29-98. 
[50] J.-C. Saut, R. Temam, Remarks on the Korteweg-de Vries equation, Israel J. Math. 24 (1976) $78-87$.

[51] A. Sjöberg, On the Korteweg-de Vries equation: Existence and uniqueness, J. Math. Anal. Appl. 29 (1970) 569-579.

[52] L. Tartar, Non linèaire et régularité, J. Funct. Anal. 9 (1972) 469-489.

[53] R. Temam, Sur un problème non linéaire, J. Math. Pures Appl. 48 (1969) 159-172.

[54] B.A. Ton, Initial boundary value problems for the Korteweg-de Vries equation, J. Differential Equations 25 (1977) $288-309$.

[55] N. Tzvetkov, Remark on the local ill-posedness for the KdV equation, C. R. Acad. Sci. Paris Sér. I Math. 329 (1999) $1043-1047$.

[56] B.-Y. Zhang, Analyticity of solutions for the generalized Korteweg-de Vries equation with respect to their initial datum, SIAM J. Math. Anal. 26 (1995) 1488-1513. 